\title{
Coupled Finite Volume Methods and Extended Finite Element Methods for the Dynamic Crack Propagation Modelling with the Pressurized Crack Surfaces
}

\author{
Shouyan Jiang and Chengbin Du \\ Department of Engineering Mechanics, Hohai University, Nanjing 210098, China \\ Correspondence should be addressed to Shouyan Jiang; syjiang@hhu.edu.cn and Chengbin Du; cbdu@hhu.edu.cn
}

Received 25 July 2017; Revised 7 October 2017; Accepted 11 October 2017; Published 28 November 2017

Academic Editor: Mickaël Lallart

Copyright (C) 2017 Shouyan Jiang and Chengbin Du. This is an open access article distributed under the Creative Commons Attribution License, which permits unrestricted use, distribution, and reproduction in any medium, provided the original work is properly cited.

\begin{abstract}
We model the fluid flow within the crack as one-dimensional flow and assume that the flow is laminar; the fluid is incompressible and accounts for the time-dependent rate of crack opening. Here, we discretise the flow equation by finite volume methods. The extended finite element methods are used for solving solid medium with crack under dynamic loads. Having constructed the approximation of dynamic extended finite element methods, the derivation of governing equation for dynamic extended finite element methods is presented. The implicit time algorithm is elaborated for the time descritisation of dominant equation. In addition, the interaction integral method is given for evaluating stress intensity factors. Then, the coupling model for modelling hydraulic fracture can be established by the extended finite element methods and the finite volume methods. We compare our present numerical results with our experimental results for verifying the proposed model. Finally, we investigate the water pressure distribution along crack surface and the effect of water pressure distribution on the fracture property.
\end{abstract}

\section{Introduction}

For hydraulic concrete structures, the external dynamic loads, such as strong earthquake, may cause cracking of these structures. The cracking of the structure causes the fluid injecting into the solid medium. The injecting fluid produces fluid pressures along crack surface and affects the deformation of the solid medium and the fracture propagation again. Many researchers [1-4] contributed to the study of hydraulic fracturing problem and these efforts led to a progressive recognition of the multiscale nature of the hydraulic fracturing problem. In simulation of hydraulic fracturing, these important aspects need to be specially concerned, namely, the flow of viscous fracturing fluid, the creation of fracture surfaces in the solid, the formation of a lag between the crack edge and the fluid front, the elastic deformation of the solid, and the leak-off of fluid from the fracture.

Some researches (2005) [5] showed that, in hydraulic structures, the water could penetrate an initiated crack, and the crack opening velocity, the magnitude of the opening, and crack mouth pressure had an important effect on the water pressure distribution. Boone and Ingraffea (1990) [6] proposed a finite difference approximation for modelling fluid flow along the fracture. Wu and Wong (2014) [7] incorporated the cubic law into the numerical manifold method for modelling fluid flow through fractures. Lisjak et al. (2017) [8] assumed that the fluid flow in discontinuous, porous rock masses was a viscous, compressible fluid, and the flow was explicitly solved based on a cubic law approximation. The fluid flow along a propagating crack surface satisfies some natural flow law. Some hypotheses [9-12], such as linear distribution of the water pressure along crack case, full reservoir pressure case, for evaluating water pressure along a propagating crack cannot reflect accurately the variation of the water pressure along new developing cracks in structures.

In recent years, many numerical methods have been developed for hydraulic fracturing modeling, such as finite element methods $[13,14]$, generalized finite element methods [15], finite-discrete element methods [8], numerical manifold 
methods [7], boundary element methods [16], discontinuous deformation analysis methods [17], and extended finite element methods (XFEM). The XFEM shows huge advantage for dealing with discontinuous problems [18-20] and also hydraulic fracture problems. The XFEM mesh does not need to align with a discontinuity. For moving discontinuities, such as crack propagation problem, it does not need to carry on remeshing. Mesh refinement is also unnecessary around a discontinuous feature. The first simulation of hydraulic fracture in XFEM was due to Réthoré et al. [21] and they developed a two-scale numerical model for fluid flow in fractured, deforming porous media. In 2009, Lecampion [22] adopted the XFEM for investigating the solution of hydraulic fracture problems. Gordeliy and Peirce (2013) [23] proposed coupled algorithms that used the XFEM to solve the elastic crack component of the elastohydrodynamic equations that governed the propagation of hydraulic fractures in an elastic medium. Subsequently, they (2013) [24] proposed two novel XFEM schemes for modeling fluid driven fractures both of which exploited an implicit level set algorithm for locating the singular free boundary that occurred when the fluid and fracture fronts coalesce. Their excellent works provide mathematical proof for using XFEM to solve hydraulic fracturing problems. Khoei et al. [25] simulated the crack growth in saturated porous media using XFEM. Taleghani [26] also developed an XFEM code to simulate fracture propagation, initiation, and intersection, and the presented coupled fluid flow-fracture mechanics simulations extended available modeling efforts and provided a unified framework for evaluating fracture design parameters and their consequences. Salimzadeh and Khalili (2015) [27] proposed a three-phase hydromechanical model for hydraulic fracturing and they handled discontinuity by using XFEM while cohesive crack model was used as fracturing criterion. Wang et al. (2015) [28] proposed a hybrid approach combining the XFEM and the finite volume method to simulate hydraulic fracturing in concrete dams. Our current study concerns developing a model for cracking modeling of structure under water pressure along a propagating crack surface and dynamic loads. Additionally, we will compare our present numerical results with our experimental results for verifying the proposed model.

This paper is organized as follows. Section 2 introduces some governing equations for elastic dynamic responses of the solid medium and fluid flow pressure within the crack. Section 3 discusses numerical approximation of extended finite element methods and finite volume methods of the flow along a crack. Section 4 gives a numerical example for investigating the water pressure distribution along crack surface and the effect of water pressure distribution on the fracture property. We also compare our present numerical results with our experimental results. Section 5 summarises the major conclusions that can be drawn from this study.

\section{Governing Equations}

2.1. Elastic Dynamic Responses of the Solid Medium. The boundary of a bounded domain, $\Omega \in R^{2}$, is partitioned into three parts: the displacement boundary $\left(\Gamma_{\mathrm{u}}\right)$, the traction boundary $\left(\Gamma_{t}\right)$, and the crack boundary $\left(\Gamma_{c}\right)$ that is tractionfree. The elastodynamic basic equation is expressed as

$$
\begin{aligned}
\nabla \cdot \boldsymbol{\sigma}+\mathbf{b} & =\rho \ddot{\mathbf{u}} \quad \text { in } \Omega, \\
\boldsymbol{\varepsilon} & =\nabla_{\mathrm{s}} \mathbf{u} \quad \text { in } \Omega, \\
\boldsymbol{\sigma} & =\mathbf{D}: \boldsymbol{\varepsilon} \quad \text { in } \Omega
\end{aligned}
$$

with the following boundary and initial conditions:

$$
\begin{aligned}
\mathbf{u}(\mathbf{x}, t) & =\overline{\mathbf{u}}(\mathbf{x}, t) \quad \text { on } \Gamma_{t}, \\
\boldsymbol{\sigma} \cdot \mathbf{n} & =\overline{\mathbf{t}} \quad \text { on } \Gamma_{\mathrm{u}}, \\
\boldsymbol{\sigma} \cdot \mathbf{n} & =0 \quad \text { on } \Gamma_{\mathrm{c}}, \\
\mathbf{u}(\mathbf{x}, t=0) & =\overline{\mathbf{u}}(0), \\
\dot{\mathbf{u}}(\mathbf{x}, t=0) & =\overline{\dot{\mathbf{u}}}(0),
\end{aligned}
$$

where $\boldsymbol{\sigma}$ is the Cauchy stress tensor, $\mathbf{b}$ is the body force vector, $\boldsymbol{\varepsilon}$ is the strain tensor, $\rho$ is the material density, $\ddot{\mathbf{u}}$ is the acceleration field vector, $\nabla_{s}$ is the symmetric part of the gradient operator, $\mathbf{u}$ is the displacement field vector, $\mathbf{D}$ is the constitutive matrix, $\mathbf{n}$ is the unit outward normal vector to the crack surface, $\overline{\mathbf{u}}$ is the prescribed displacement, $\overline{\mathbf{t}}$ is the external traction vector, $\overline{\mathbf{u}}(0)$ is the initial displacement vector, and $\overline{\dot{\mathbf{u}}}(0)$ is the initial velocity vector.

2.2. Fluid Flow Pressure within the Crack. In this paper, we model the fluid flow within the crack as one-dimensional flow. Assume that the flow is laminar and the fluid is incompressible. But here we account for the time-dependent rate of crack opening.

The conservation of the incompressible fluid in the fracture can be expressed as [6]

$$
\nabla \cdot \mathbf{q}+\dot{w}+g=0
$$

where $\nabla$ is the divergence operator defined in $x$ direction; $\mathbf{q}$ is the fluid flux; $\dot{w}$ is the time-dependent rate of crack opening, and $\dot{w}=\partial w / \partial t$; and $g$ is the fluid loss into the solid media, and here we ignore the fluid loss; that is, $g=0$.

Additionally, Poiseuille's law [29] gives the following expression:

$$
\mathbf{q}=-\frac{w^{3}}{12 \mu} \nabla p,
$$

where $w$ is the crack opening; $\mu$ is the fluid viscosity; and $p$ is the fluid pressure.

The pressure boundary conditions at the fluid injection point in the crack are

$$
p(x=0, t)=p_{0},
$$

where $p_{0}$ is the pressure of the fluid injection point.

In the fluid lag zone [30],

$$
p(x, t)<0 \quad\left(l_{\mathrm{t}} \leq x \leq l\right) .
$$

According to the fluid pressure continuity, lag condition (6) provides the net-pressure boundary condition at the fluid front $x=l_{\mathrm{t}}$ for the fluid flow equations (3). 

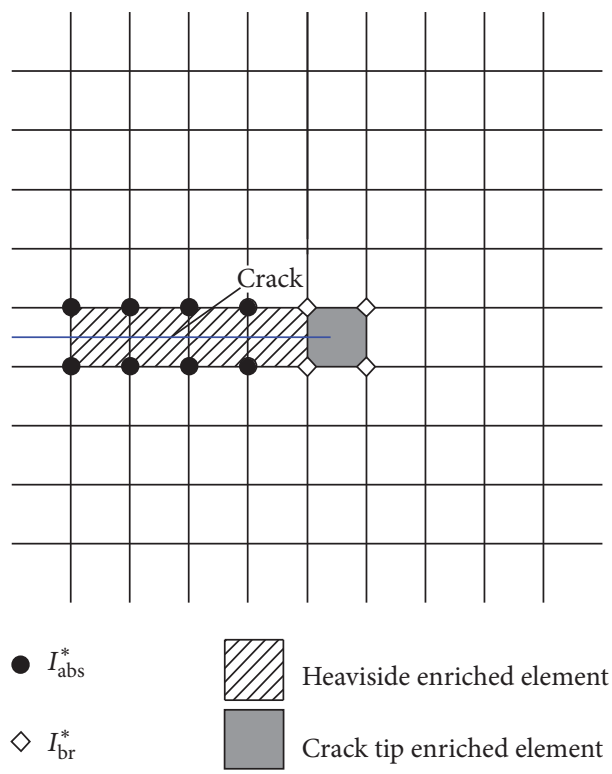

FIGURE 1: A strategy for enriched elements and nodes.

\section{Numerical Approximation}

\subsection{XFEM for Dynamic Problems}

3.1.1. XFEM Approximation. The XFEM approximation for $2 \mathrm{D}$ cracked domains can be written as

$$
\begin{aligned}
\mathbf{u}^{\mathrm{h}}(\mathbf{x})= & \sum_{i \in I} N_{i}(\mathbf{x}) \mathbf{u}_{i}+\sum_{i \in I_{\mathrm{abs}}^{*}} N_{i}^{*}(\mathbf{x})\left[H(\mathbf{x})-H\left(\mathbf{x}_{i}\right)\right] \mathbf{a}_{i} \\
& +\sum_{i \in I_{\mathrm{br}}^{*}} N_{i}^{*}(\mathbf{x}) \sum_{j=1}^{4}\left[F_{j}(\mathbf{x})-F_{j}\left(\mathbf{x}_{i}\right)\right] \mathbf{b}_{i}^{j},
\end{aligned}
$$

where $N_{i}(\mathbf{x})$ is the standard finite element shape function of node $i ; \mathbf{u}_{i}$ is the unknown of the standard finite element part at node $i ; I$ is the set of all nodes in the domain; and $N_{i}^{*}(\mathbf{x})$ is the partition of unity functions, and the function can hold the same form with the standard finite element shape function but is not necessary; $\mathbf{a}_{i}$ and $\mathbf{b}_{i}^{j}$ are the nodal enriched degree of freedom; $I_{\mathrm{abs}}^{*}$ and $I_{\mathrm{br}}^{*}$ are the set of enrichment nodes shown in Figure 1 , and $I_{\mathrm{abs}}^{*}, I_{\mathrm{br}}^{*} \subset I$.

For these elements that are cut completely by a crack, the nodes of these elements that are the nodal subset $I_{\mathrm{abs}}^{*}$ are enriched by Heaviside function $H(\mathbf{x})$. The definition of Heaviside function $H(\mathbf{x})$ follows:

$$
H(\mathbf{x})= \begin{cases}+1, & \left(\mathbf{x}-\mathbf{x}^{*}\right) \cdot \mathbf{n}>0 \\ -1, & \left(\mathbf{x}-\mathbf{x}^{*}\right) \cdot \mathbf{n}<0\end{cases}
$$

where $\mathbf{x}^{*}$ is the projection of a point $\mathbf{x}$ on the crack surface; $\mathbf{n}$ is the unit outward normal to the crack surface.

For these elements that are cut partially by a crack, the nodes of these elements that are the nodal subset $I_{\mathrm{br}}^{*}$ are enriched by the crack tip enrichment function $F_{j}(\mathbf{x})$. The definition of the crack tip enrichment function $F_{j}(\mathbf{x})$ follows:

$$
\begin{aligned}
& F_{j=1,2,3,4}(r, \theta) \\
& =\left\{\sqrt{r} \sin \frac{\theta}{2}, \sqrt{r} \cos \frac{\theta}{2}, \sqrt{r} \sin \theta \sin \frac{\theta}{2}, \sqrt{r} \sin \theta \cos \frac{\theta}{2}\right\},
\end{aligned}
$$

where $r$ and $\theta$ are the local crack tip coordinate system.

3.1.2. Discrete Equations. By the principle of virtual work, the following discrete equations can be obtained:

$$
\mathbf{M} \ddot{\mathbf{u}}^{\mathrm{h}}+\mathbf{K} \mathbf{u}^{\mathrm{h}}=\mathbf{f},
$$

where $\mathbf{K}(\mathbf{M})$ is the global stiffness (mass) matrix assembled by the element stiffness (mass) matrix; $\mathbf{f}$ is the global external force vector; $\mathbf{u}^{\mathrm{h}}$ and $\ddot{\mathbf{u}}^{\mathrm{h}}$ denote the vector of nodal parameters (which include the classic degrees of freedom, $\mathbf{u}$, and the enrichment degrees of freedom, a, b) and its second time derivative, respectively; and

$$
\begin{aligned}
\mathbf{u}^{\mathrm{h}} & =\{\mathbf{u}, \mathbf{a}, \quad \mathbf{b}\}^{\mathrm{T}}, \\
\ddot{\mathbf{u}}^{\mathrm{h}} & =\{\ddot{\mathbf{u}}, \ddot{\mathbf{a}}, \ddot{\mathbf{b}}\}^{\mathrm{T}} .
\end{aligned}
$$

The element stiffness matrix is expressed by

$$
\mathbf{k}^{\mathrm{e}}=\left[\begin{array}{ccc}
\mathbf{k}^{\mathrm{uu}} & \mathbf{k}^{\mathrm{ua}} & \mathbf{k}^{\mathrm{ub}} \\
\mathbf{k}^{\mathrm{au}} & \mathbf{k}^{\mathrm{aa}} & \mathbf{k}^{\mathrm{ab}} \\
\mathbf{k}^{\mathrm{bu}} & \mathbf{k}^{\mathrm{ba}} & \mathbf{k}^{\mathrm{bb}}
\end{array}\right]
$$


where

$$
\begin{aligned}
& \mathbf{k}^{r s}=\int_{\Omega^{e}}\left(\mathbf{B}^{r}\right)^{\mathrm{T}} \mathbf{D} \mathbf{B}^{s} \mathrm{~d} \Omega \\
& =\int_{\Omega^{\mathrm{e}}}\left[\begin{array}{llll}
\mathbf{B}_{1}^{r} & \mathbf{B}_{2}^{r} & \mathbf{B}_{3}^{r} & \mathbf{B}_{4}^{r}
\end{array}\right]^{\mathrm{T}} \mathbf{D}\left[\begin{array}{llll}
\mathbf{B}_{1}^{s} & \mathbf{B}_{2}^{s} & \mathbf{B}_{3}^{s} & \mathbf{B}_{4}^{s}
\end{array}\right] \mathrm{d} \Omega \\
& (r, s=\mathrm{u}, \mathrm{a}, \mathrm{b}), \\
& \mathbf{B}_{i}^{\mathrm{u}}=\left[\begin{array}{ccc}
\frac{\partial N_{i}}{\partial x} & 0 & \frac{\partial N_{i}}{\partial y} \\
0 & \frac{\partial N_{i}}{\partial y} & \frac{\partial N_{i}}{\partial x}
\end{array}\right]^{\mathrm{T}}, \quad i=1,2,3,4 \\
& \mathbf{B}_{i}^{\mathrm{a}}=\left[\begin{array}{ccc}
\frac{\partial\left(N_{i}^{*} \widehat{H}\right)}{\partial x} & 0 & \frac{\partial\left(N_{i}^{*} \widehat{H}\right)}{\partial y} \\
0 & \frac{\partial\left(N_{i}^{*} \widehat{H}\right)}{\partial y} & \frac{\partial\left(N_{i}^{*} \widehat{H}\right)}{\partial x}
\end{array}\right]^{\mathrm{T}}, \\
& \widehat{H}=H(\mathbf{x})-H\left(\mathbf{x}_{i}\right), i=1,2,3,4, \\
& \mathbf{B}_{i}^{\mathrm{b}}=\left[\begin{array}{llll}
\mathbf{B}_{i}^{\mathrm{b} 1} & \mathbf{B}_{i}^{\mathrm{b} 2} & \mathbf{B}_{i}^{\mathrm{b} 3} & \mathbf{B}_{i}^{\mathrm{b} 4}
\end{array}\right], \\
& \mathbf{B}_{i}^{\mathrm{bj}}=\left[\begin{array}{ccc}
\frac{\partial\left(N_{i}^{*} \widehat{F}_{j}\right)}{\partial x} & 0 & \frac{\partial\left(N_{i}^{*} \widehat{F}_{j}\right)}{\partial y} \\
0 & \frac{\partial\left(N_{i}^{*} \widehat{F}_{j}\right)}{\partial y} & \frac{\partial\left(N_{i}^{*} \widehat{F}_{j}\right)}{\partial x}
\end{array}\right]^{\mathrm{T}}, \\
& \widehat{F}_{j}=F_{j}(\mathbf{x})-F_{j}\left(\mathbf{x}_{i}\right), i, j=1,2,3,4 .
\end{aligned}
$$

The element mass matrix is expressed by

$$
\mathbf{m}^{\mathrm{e}}=\left[\begin{array}{ccc}
\mathbf{m}^{\mathrm{uu}} & \mathbf{m}^{\mathrm{ua}} & \mathbf{m}^{\mathrm{ub}} \\
\mathbf{m}^{\mathrm{au}} & \mathbf{m}^{\mathrm{aa}} & \mathbf{m}^{\mathrm{ab}} \\
\mathbf{m}^{\mathrm{bu}} & \mathbf{m}^{\mathrm{ba}} & \mathbf{m}^{\mathrm{bb}}
\end{array}\right],
$$

where

$$
\begin{aligned}
& \mathbf{m}^{\mathrm{uu}}=\int_{\Omega^{\mathrm{e}}} \rho(\mathbf{N})^{\mathrm{T}} \mathbf{N} d \Omega, \\
& \mathbf{m}^{\mathrm{ua}}=\left[\mathbf{m}^{\mathrm{au}}\right]^{\mathrm{T}}=\int_{\Omega^{\mathrm{e}}} \rho(\mathbf{N})^{\mathrm{T}}(\mathbf{N} \widehat{H}) d \Omega, \\
& \mathbf{m}_{j}^{\mathrm{ub}}=\left[\mathbf{m}_{j}^{\mathrm{bu}}\right]^{\mathrm{T}}=\int_{\Omega^{\mathrm{e}}} \rho(\mathbf{N})^{\mathrm{T}}\left(\mathbf{N} \widehat{F}_{j}\right) d \Omega, \\
& \mathbf{m}^{\mathrm{aa}}=\int_{\Omega^{\mathrm{e}}} \rho(\mathbf{N} \widehat{H})^{\mathrm{T}}(\mathbf{N} \widehat{H}) d \Omega, \\
& \mathbf{m}_{j}^{\mathrm{ab}}=\left[\mathbf{m}_{j}^{\mathrm{ba}}\right]^{\mathrm{T}}=\int_{\Omega^{\mathrm{e}}} \rho(\mathbf{N} \widehat{H})^{\mathrm{T}}\left(\mathbf{N} \widehat{F}_{j}\right) d \Omega, \\
& \mathbf{m}_{j k}^{\mathrm{bb}}=\int_{\Omega^{\mathrm{e}}} \rho\left(\mathbf{N} \widehat{F}_{j}\right)^{\mathrm{T}}\left(\mathbf{N} \widehat{F_{k}}\right) d \Omega,
\end{aligned}
$$$$
j, k=1,2,3,4,
$$

$$
\begin{aligned}
\mathbf{N} & =\left[\begin{array}{llll}
\mathbf{N}_{1} & \mathbf{N}_{2} & \mathbf{N}_{3} & \mathbf{N}_{4}
\end{array}\right], \\
\mathbf{N}_{i} & =\left[\begin{array}{cc}
N_{i} & 0 \\
0 & N_{i}
\end{array}\right], \quad i=1,2,3,4 .
\end{aligned}
$$

The element external force vector is

$$
\mathbf{f}^{\mathrm{e}}=\left[\begin{array}{lll}
\mathbf{f}^{\mathrm{u}} & \mathbf{f}^{\mathrm{a}} & \mathbf{f}^{\mathrm{b}}
\end{array}\right]^{\mathrm{T}},
$$

where

$$
\begin{aligned}
\mathbf{f}^{\mathrm{u}}= & \int_{\Omega^{\mathrm{e}}} \mathbf{N}^{\mathrm{T}} \mathbf{b} \mathrm{d} \Omega+\int_{\Gamma_{\mathrm{t}}^{e}} \mathbf{N}^{\mathrm{T}} \overline{\mathbf{t}} \mathrm{d} \Gamma, \\
\mathbf{f}^{\mathrm{a}}= & \int_{\Omega^{\mathrm{e}}}\left(\mathbf{N}^{*} \widehat{\mathbf{H}}\right)^{\mathrm{T}} \mathbf{b} \mathrm{d} \Omega+\int_{\Gamma_{\mathrm{t}}^{\mathrm{e}}}\left(\mathbf{N}^{*} \widehat{\mathbf{H}}\right)^{\mathrm{T}} \overline{\mathbf{t}} \mathrm{d} \Gamma \\
& +\int_{\Gamma_{\mathrm{c}}^{e}}\left(\mathbf{N}^{*} \widehat{\mathbf{H}}\right)^{\mathrm{T}}(\mathbf{p} \cdot \mathbf{n}) \mathrm{d} \Gamma, \\
\mathbf{f}^{\mathrm{b} j}= & \int_{\Omega^{e}}\left(\mathbf{N}^{*} \widehat{\mathbf{F}}_{j}\right)^{\mathrm{T}} \mathbf{b} \mathrm{d} \Omega+\int_{\Gamma_{\mathbf{t}}^{e}}\left(\mathbf{N}^{*} \widehat{\mathbf{F}}_{j}\right)^{\mathrm{T}} \overline{\mathbf{t}} \mathrm{d} \Gamma \\
& +\int_{\Gamma_{c}^{e}}\left(\mathbf{N}^{*} \widehat{\mathbf{F}}_{j}\right)^{\mathrm{T}}(\mathbf{p} \cdot \mathbf{n}) \mathrm{d} \Gamma, \quad j=1,2,3,4 .
\end{aligned}
$$

3.1.3. Time Integration Schemes. The following time-integration scheme is used in dynamic analysis. Equation (10) for a specific time $t+\Delta t$ is expressed as

$$
\begin{aligned}
\mathbf{M} \ddot{\mathbf{u}}_{t+\Delta t}+\mathbf{K} \mathbf{u}_{t+\Delta t}= & \mathbf{f}_{t+\Delta t}, \\
\dot{\mathbf{u}}_{t+\Delta t}= & \dot{\mathbf{u}}_{t}+(1-\gamma) \ddot{\mathbf{u}}_{t} \Delta t+\gamma \ddot{\mathbf{u}}_{t+\Delta t} \Delta t \\
\mathbf{u}_{t+\Delta t}= & \mathbf{u}_{t}+\dot{\mathbf{u}}_{t} \Delta t+\left(\frac{1}{2}-\beta\right) \ddot{\mathbf{u}}_{t} \Delta t^{2} \\
& +\beta \ddot{\mathbf{u}}_{t+\Delta t} \Delta t^{2}
\end{aligned}
$$

where $\mathbf{u}_{t}, \dot{\mathbf{u}}_{t}$, and $\ddot{\mathbf{u}}_{t}$ are the displacement, velocity, and acceleration vectors at time $t$, respectively; $\Delta t$ is the time step; $\gamma$ and $\beta$ are parameters that can be determined to obtain integration accuracy and stability, with

$$
\begin{aligned}
\beta & =\frac{1}{4}(1-\alpha)^{2}, \\
\gamma & =\frac{1}{2}-\alpha, \\
-\frac{1}{3} & \leq \alpha \leq 0 .
\end{aligned}
$$

Here, referring to the software ABAQUS (ABAQUS Theory Manual, Version 6.9), we set parameter $\alpha=-0.05$ to remove the slight high frequency noise in the solution without having any significant effect on the meaningful, lower frequency response.

The following steps describe the prescribe integration method procedure, while neglecting the damping effects. 

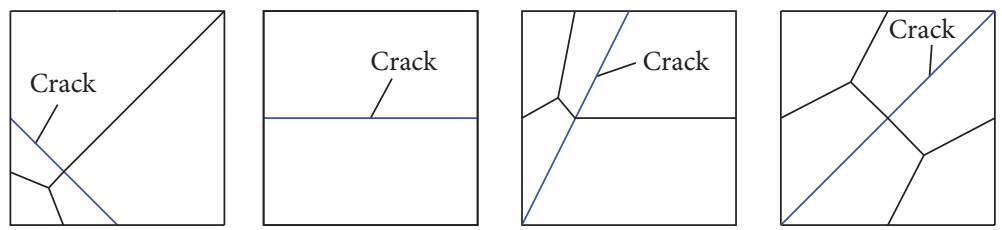

(a) Elements partitioned completely by a straight crack
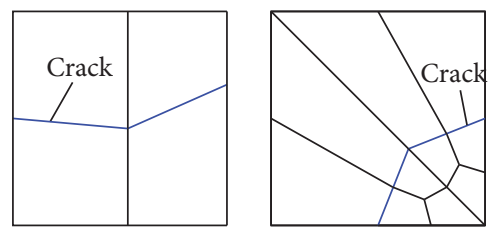

(b) Elements partitioned completely by a broken crack
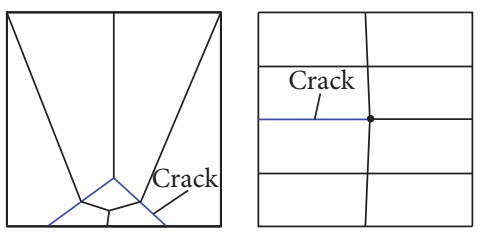

(c) Elements partitioned partially by a straight crack

FIGURE 2: Element partitioning method for these elements containing a discontinuous interface.

\section{(I) Initial Calculations}

(i) Form stiffness matrix $\mathbf{K}$, and mass matrix $\mathbf{M}$.

(ii) Give the initial displacement vector $\mathbf{u}_{0}$ and the initial velocity vector $\dot{\mathbf{u}}_{0}$. Then, calculate the initial acceleration vector $\ddot{\mathbf{u}}_{0}$ by the equilibrium equation:

$$
\mathbf{M} \ddot{\mathbf{u}}_{0}+\mathbf{K} \mathbf{u}_{0}=\mathbf{f}_{0} .
$$

(iii) Select a time step $\Delta t$ and the parameters $\beta$ and $\gamma$. Here, $\beta=0.275625$ and $\gamma=0.55$ are used. Calculate integration constants:

$$
\begin{aligned}
& c_{0}=\frac{1}{\beta \Delta t^{2}}, \\
& c_{1}=\frac{\gamma}{\beta \Delta t}, \\
& c_{2}=\frac{1}{\beta \Delta t}, \\
& c_{3}=\frac{1}{2 \beta}-1, \\
& c_{4}=\frac{\gamma}{\beta}-1, \\
& c_{5}=\Delta t\left(\frac{\gamma}{2 \beta}-1\right), \\
& c_{6}=\Delta t(1-\gamma), \\
& c_{7}=\gamma \Delta t .
\end{aligned}
$$

(iv) Form the effective stiffness matrix $\widetilde{\mathbf{K}}$ :

$$
\widetilde{\mathbf{K}}=\mathbf{K}+c_{0} \mathbf{M}
$$

(II) For Each Time Step

(i) Calculate effective loads $\widetilde{\mathbf{f}}_{t+\Delta t}$ at time $t+\Delta t$ :

$$
\widetilde{\mathbf{f}}_{t+\Delta t}=\mathbf{f}_{t+\Delta t}+\mathbf{M}\left(c_{0} \mathbf{u}_{t}+c_{2} \dot{\mathbf{u}}_{t}+c_{3} \ddot{\mathbf{u}}_{t}\right) .
$$

(ii) Solve for the displacement vector $\mathbf{u}_{t+\Delta t}$ at time $t+\Delta t$ :

$$
\widetilde{\mathbf{K}} \mathbf{u}_{t+\Delta t}=\widetilde{\mathbf{f}}_{t+\Delta t} .
$$

(iii) Calculate the acceleration vector $\ddot{\mathbf{u}}_{t+\Delta t}$ and the velocity vector $\dot{\mathbf{u}}_{t+\Delta t}$ at time $t+\Delta t$ :

$$
\begin{aligned}
& \ddot{\mathbf{u}}_{t+\Delta t}=c_{0}\left(\mathbf{u}_{t+\Delta t}-\mathbf{u}_{t}\right)-c_{2} \dot{\mathbf{u}}_{t}-c_{3} \ddot{\mathbf{u}}_{t}, \\
& \dot{\mathbf{u}}_{t+\Delta t}=\dot{\mathbf{u}}_{t}+c_{6} \ddot{\mathbf{u}}_{t}+c_{7} \ddot{\mathbf{u}}_{t+\Delta t} .
\end{aligned}
$$

3.1.4. Integration Schemes at the Discontinuities. For these elements partitioned by a crack, the ordinary Gauss quadrature rules cannot accurately calculate the integration of enrichment function. An alternative method that is dividing the enrichment element into a set of subpolygons usually needs to be used [31]. Additionally, some simplified numerical integration methods also had been proposed in literatures. Ventura [32] conducted an important first attempt to simplify numerical integration. His work is based on replacing nonpolynomial functions by "equivalent" polynomials. However, the proposed method is exact for triangular and tetrahedral elements, but for quadrilateral elements, when the opposite sides are not parallel, additional approximation is introduced. Another method is strain smoothing [33]. In strain smoothing, the surface integration is transformed into equivalent boundary integration by use of the GreenOstrogradsky theorem. Natarajan et al. [34] used the new numerical integration proposed for arbitrary polygons [35] to integrate the discontinuous and singular integrands appearing in the XFEM stiffness matrix. In this paper, the method subdividing the element into subquads is used. For these elements partitioned completely or partially by a crack, the method subdividing these elements into subquads is shown in Figure 2.

To solve the element stiffness or mass matrix of these enrichment elements, each subquad element is, respectively, transferred into the standard element $(-1,1) \times(-1,1)$ by the method of the coordinate transformation. The Gauss integration points are distributed into each subquad. To improve the accuracy of crack tip integration, 15 Gauss integration points 


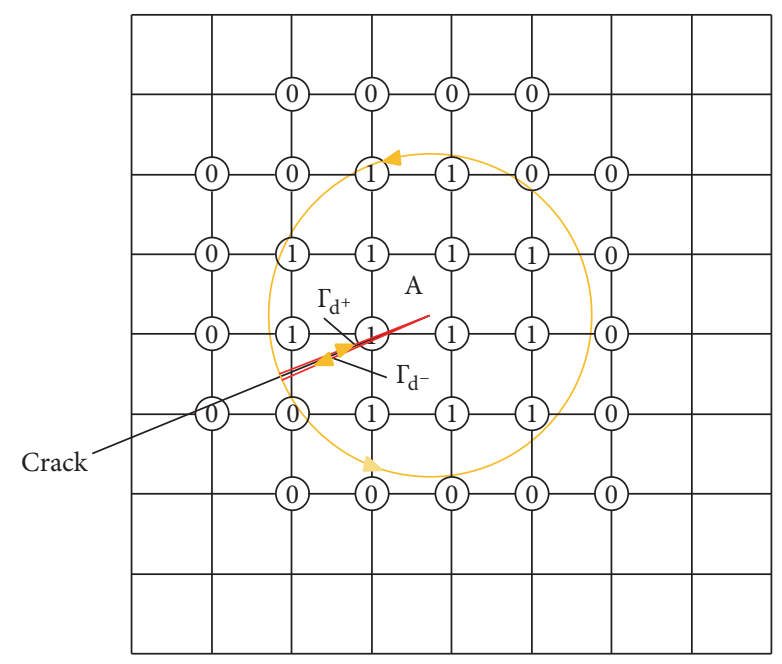

FIGURE 3: Elements selection for the interaction integral near the crack tip.

are distributed into each subquad for elements containing crack tip. The numerical integration is firstly performed in each subquad element domain, and then the element stiffness or mass matrix of the enrichment element can be obtained by assembling the numerical integration results of each subquad element. It is worthwhile pointing out that these subquads are only necessary for integration purposes. They do not provide additional degrees of freedom for the global stiffness and mass matrix.

\subsubsection{Interaction Integral for Computing Stress Intensity Fac-} tors. Take field $1,\left(\sigma_{i j}^{(1)}, \varepsilon_{i j}^{(1)}, u_{i}^{(1)}\right)$, for the actual field, and field $2,\left(\sigma_{i j}^{(2)}, \varepsilon_{i j}^{(2)}, u_{i}^{(2)}\right)$, for the auxiliary field. The actual field is obtained from numerical solutions computed by using XFEM, and the auxiliary field refers to the asymptotic results of linear elastic fracture dynamics [36]. The interaction integral equation which is used to evaluate the stress intensity factors is as follows:

$$
\begin{aligned}
& I^{(1,2)}=\int_{\mathrm{A}}\left(\sigma_{i j}^{(1)} u_{i, 1}^{(2)}+\sigma_{i j}^{(2)} u_{i, 1}^{(1)}-\frac{1}{2} \sigma_{i k}^{(1)} \varepsilon_{i k}^{(2)} \delta_{1 j}\right) q_{, j} d \Omega \\
& +\int_{\Gamma_{\mathrm{d}^{+}} \cup \Gamma_{\mathrm{d}^{-}}}\left(\frac{1}{2} \sigma_{i k}^{(1)} \varepsilon_{i k}^{(2)} \delta_{1 j}-\sigma_{i j}^{(1)} u_{i, 1}^{(2)}-\sigma_{i j}^{(2)} u_{i, 1}^{(1)}\right) \\
& \cdot q n_{j} \mathrm{~d} \Gamma .
\end{aligned}
$$

The second term in (26) denotes the contribution of traction along crack interface. As shown in Figure 3, A denotes the circle domain with centre at the crack tip and the radius $R ; \Gamma_{\mathrm{d}^{+}} \cup \Gamma_{\mathrm{d}^{-}}$consists of a interface starting from the external integration radius to crack tip in a two-way manner. $R$ is defined as

$$
R=r_{\mathrm{k}} h_{\mathrm{e}}
$$

where $h_{\mathrm{e}}$ is the crack-tip element size; $r_{\mathrm{k}}$ is a user-specified scalar multiple; $q$ is the weight function; $q=1$ if the node lies in $\mathrm{A}$; and $q=0$ if the node lies outside of $\mathrm{A}$ or lies on the boundary of A. The weight function $q$ in the interior of an element is obtained by the interpolation of the nodal value:

$$
q=\sum_{i=1}^{4} N_{i} q_{i}
$$

Additionally, the interaction integral relates to the stress intensity factors through the relation:

$$
M^{(1,2)}=\frac{2}{E^{*}}\left[K_{\mathrm{I}} K_{\mathrm{I}}^{\mathrm{aux}}+K_{\mathrm{II}} K_{\mathrm{II}}^{\mathrm{aux}}\right],
$$

where $K_{\mathrm{I}}^{\mathrm{aux}}$ and $K_{\mathrm{II}}^{\mathrm{aux}}$ are the local auxiliary stress intensity factors for the auxiliary fields, respectively; and the definition of $E^{*}$ is

$$
E^{*}= \begin{cases}E & (\text { plane stress) } \\ \frac{E}{1-v^{2}} & \text { (plane strain). }\end{cases}
$$

By setting $K_{\mathrm{I}}^{\mathrm{aux}}=1$ and $K_{\mathrm{II}}^{\mathrm{aux}}=0$ as well as $M^{(1,2)}=$ $M_{1}^{(1,2)}$, we obtain the expression of $K_{\mathrm{I}}$ as follows:

$$
K_{\mathrm{I}}=\frac{E^{*} M_{1}^{(1,2)}}{2} \text {. }
$$

Similarly, we obtain the equality

$$
K_{\mathrm{II}}=\frac{E^{*} M_{2}^{(1,2)}}{2}
$$

by setting $K_{\mathrm{I}}^{\mathrm{aux}}=0$ and $K_{\mathrm{II}}^{\mathrm{aux}}=1$ and $M^{(1,2)}=M_{2}^{(1,2)}$.

3.1.6. Crack Propagation Criteria. The maximum circumferential stress criterion [37] is used to determine the crack growth direction. Once $K_{\mathrm{I}}$ and $K_{\mathrm{II}}$ are calculated, the criterion gives the following crack growth direction:

$$
\theta_{\mathrm{c}}=2 \tan ^{-1} \frac{1}{4}\left(\frac{K_{\mathrm{I}}}{K_{\mathrm{II}}} \pm \sqrt{\left(\frac{K_{\mathrm{I}}}{K_{\mathrm{II}}}\right)^{2}+8}\right),
$$

where $\theta_{c}$ is the crack growth angle in the local crack-tip coordinate system. If $K_{\mathrm{II}}=0$, then $\theta_{\mathrm{c}}=0$. It should also be noted that if $K_{\mathrm{II}}>0$, the crack growth angle $\theta_{\mathrm{c}}<0$, and if $K_{\mathrm{II}}<0$, then $\theta_{\mathrm{c}}>0$. Sukumar and Prévost (2003) [38] proposed a computationally more amenable expression for $\theta_{\mathrm{c}}$ :

$$
\theta_{\mathrm{c}}=2 \tan ^{-1}\left(\frac{-2 K_{\mathrm{II}} / K_{\mathrm{I}}}{1+\sqrt{1+8\left(K_{\mathrm{II}} / K_{\mathrm{I}}\right)^{2}}}\right) .
$$

The equivalent stress intensity factor then follows:

$$
K_{\mathrm{e}}=\cos \frac{\theta_{\mathrm{c}}}{2}\left(K_{\mathrm{I}} \cos ^{2} \frac{\theta_{\mathrm{c}}}{2}-1.5 K_{\mathrm{II}} \sin \theta_{\mathrm{c}}\right) .
$$

The double- $K$ criterion [39] is used for determining crack propagation. The crack propagation processes can be expressed as follows: 


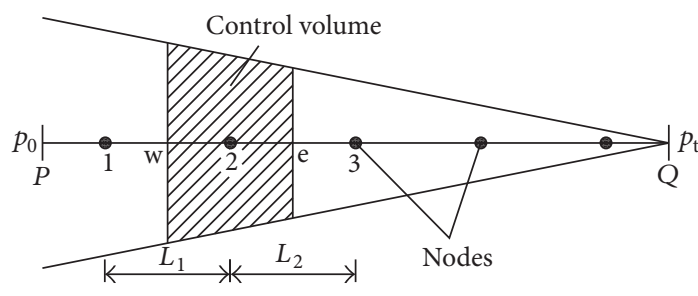

FIGURE 4: Dividing fluid domain into discrete control volumes.

(i) If $K_{\mathrm{e}}<K_{\mathrm{Ic}}^{\mathrm{ini}}$, the crack does not propagate.

(ii) When $K_{\mathrm{e}}=K_{\mathrm{Ic}}^{\mathrm{ini}}$, the performed crack begins to crack initially.

(iii) When $K_{\mathrm{Ic}}^{\text {ini }}<K_{\mathrm{e}}<K_{\mathrm{Ic}}^{\text {un }}$, the propagating crack develops steadily.

(iv) When $K_{\mathrm{e}} \geq K_{\mathrm{Ic}}^{\mathrm{un}}$, the crack propagates unsteadily.

$K_{\mathrm{Ic}}^{\mathrm{ini}}$ is the initiation toughness; $K_{\mathrm{Ic}}^{\mathrm{un}}$ is the unstable fracture toughness.

3.2. Finite Volume Methods of the Flow along a Crack. Considering (3) and (4) and ignoring the fluid loss into the solid media, the following expression can be obtained:

$$
\frac{\partial}{\partial x}\left(k \frac{\partial p}{\partial x}\right)+\frac{\partial w}{\partial t}=0
$$

where

$$
k=-\frac{w^{3}}{12 \mu} .
$$

3.2.1. Discretised Equation. Equation (36) is one-dimensional steady state diffusion equation. Here, we discretise the equation by finite volume methods. The first step in the finite volume method is to divide the domain into discrete control volumes. As shown in Figure 4, we place a number of nodes in the fluid space between $P$ and $Q$. The boundaries of control volumes are positioned midway between adjacent nodes. Thus, each node is surrounded by a control volume. The west side face of the control volume is referred to by " $w$ " and the east side face of the control volume is referred to by "e."

Integrating the governing equation in (36) over a control volume, the following discretised equation can be obtained.

$$
\begin{aligned}
\int_{\Delta V} & \frac{\partial}{\partial x}\left(k \frac{\partial p}{\partial x}\right) \mathrm{d} V+\int_{\Delta V} \frac{\partial w}{\partial t} \mathrm{~d} V \\
= & \oint_{A} n\left(k \frac{\partial p}{\partial x}\right) \mathrm{d} A+\int_{\Delta V} \frac{\partial w}{\partial t} \mathrm{~d} V \\
= & \left(k A \frac{\partial p}{\partial x}\right)_{\mathrm{e}}-\left(k A \frac{\partial p}{\partial x}\right)_{\mathrm{w}}+\int_{\Delta V} \frac{\partial w}{\partial t} \mathrm{~d} V \\
= & \left(k A \frac{\partial p}{\partial x}\right)_{\mathrm{e}}-\left(k A \frac{\partial p}{\partial x}\right)_{\mathrm{w}}+\frac{\Delta \bar{w}}{\Delta t} \cdot \Delta V=0,
\end{aligned}
$$

where $A$ is the cross-sectional area of the control volume face; $\Delta V$ is the volume; $\bar{w}$ is the average crack opening width over a control volume.
According to (36) and observing a typical finite volume element with node 2 , we can obtain

$$
\left(k A \frac{\partial p}{\partial x}\right)_{\mathrm{e}}-\left(k A \frac{\partial p}{\partial x}\right)_{\mathrm{w}}=-\frac{\Delta \bar{w}_{2}}{\Delta t} \cdot \Delta V_{2} .
$$

To calculate gradients at the control volume faces, a linear approximate distribution of pressure is considered here. So (39) yields

$$
k_{\mathrm{e}} A_{\mathrm{e}} \frac{p_{3}-p_{2}}{L_{2}}-k_{\mathrm{w}} A_{\mathrm{w}} \frac{p_{2}-p_{1}}{L_{1}}=-\frac{\Delta \bar{w}_{2}}{\Delta t} \cdot \Delta V_{2} .
$$

$k_{\mathrm{w}}, A_{\mathrm{w}}, k_{\mathrm{e}}$, and $A_{\mathrm{e}}$ can be obtained by the way of linearly interpolated values, and

$$
\begin{aligned}
& k_{\mathrm{w}}=\delta_{\mathrm{w}} k_{1}+\left(1-\delta_{\mathrm{w}}\right) k_{2}, \\
& A_{\mathrm{w}}=\delta_{\mathrm{w}} A_{1}+\left(1-\delta_{\mathrm{w}}\right) A_{2}, \\
& \qquad \delta_{\mathrm{w}}=\frac{L_{\mathrm{w} 2}}{L_{1}}, \\
& k_{\mathrm{e}}=\delta_{\mathrm{e}} k_{2}+\left(1-\delta_{\mathrm{e}}\right) k_{3}, \\
& A_{\mathrm{e}}=\delta_{\mathrm{e}} A_{2}+\left(1-\delta_{\mathrm{e}}\right) A_{3},
\end{aligned}
$$

Equation (40) can be rewritten as

$$
a_{21} p_{1}+a_{22} p_{2}+a_{23} p_{3}=b_{2}
$$

where

$$
\begin{aligned}
& a_{21}=\frac{k_{\mathrm{w}} A_{\mathrm{w}}}{L_{1}}, \\
& a_{22}=-\frac{k_{\mathrm{w}} A_{\mathrm{w}}}{L_{1}}-\frac{k_{\mathrm{e}} A_{\mathrm{e}}}{L_{2}}, \\
& a_{23}=\frac{k_{\mathrm{e}} A_{\mathrm{e}}}{L_{2}}, \\
& b_{2}=-\frac{\Delta \bar{w}_{2}}{\Delta t} \cdot \Delta V_{2} .
\end{aligned}
$$

\subsubsection{Boundary Conditions}

(I) Boundary Conditions for Fluid Injection Point. As shown in Figure 5, integration of (36) over the control volume surrounding node 1 gives

$$
k_{\mathrm{e}} A_{\mathrm{e}} \frac{p_{2}-p_{1}}{L_{1}}-k_{\mathrm{w}} A_{\mathrm{w}} \frac{p_{1}-p_{\mathrm{w}}}{L_{0}}=-\frac{\Delta \bar{w}_{1}}{\Delta t} \cdot \Delta V_{1} .
$$

Equation (44) can be rewritten as

$$
a_{11} p_{1}+a_{12} p_{2}=b_{1},
$$




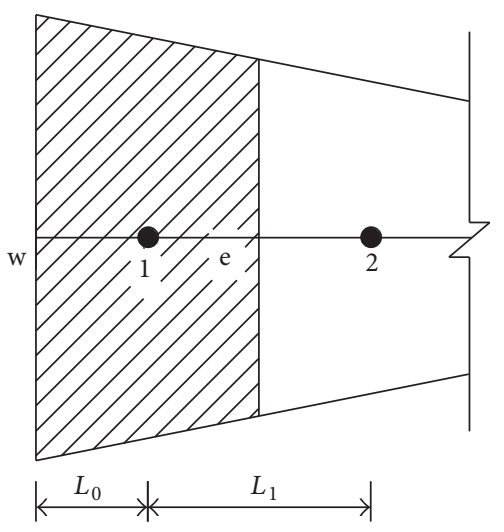

FIGURE 5: Boundary conditions for fluid injection point.

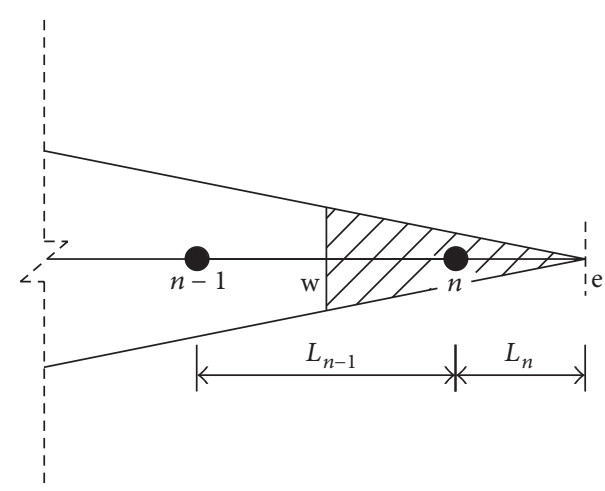

Figure 6: Boundary conditions for fluid front.

where

$$
\begin{aligned}
a_{11} & =-\frac{k_{\mathrm{e}} A_{\mathrm{e}}}{L_{1}}-\frac{k_{\mathrm{w}} A_{\mathrm{w}}}{L_{0}}, \\
a_{12} & =\frac{k_{\mathrm{e}} A_{\mathrm{e}}}{L_{1}}, \\
b_{1} & =-\frac{\Delta \bar{w}_{1}}{\Delta t} \cdot \Delta V_{1}-\frac{k_{\mathrm{w}} A_{\mathrm{w}}}{L_{0}} p_{\mathrm{w}}, \\
k_{\mathrm{e}} & =\delta_{\mathrm{e}} k_{1}+\left(1-\delta_{\mathrm{e}}\right) k_{2}, A_{\mathrm{e}}=\delta_{\mathrm{e}} A_{1}+\left(1-\delta_{\mathrm{e}}\right) A_{2} .
\end{aligned}
$$

$k_{\mathrm{w}}$ and $A_{\mathrm{w}}$ can be calculated directly according to crack opening displacement, and $p_{\mathrm{w}}$ is the pressure of the fluid injection point.

(II) Boundary Conditions for Fluid Front. Similarly, as shown in Figure 6, integration of (36) over the control volume surrounding node $n$ gives

$$
k_{\mathrm{e}} A_{\mathrm{e}} \frac{p_{\mathrm{t}}-p_{n}}{L_{n}}-k_{\mathrm{w}} A_{\mathrm{w}} \frac{p_{n}-p_{n-1}}{L_{n-1}}=-\frac{\Delta \bar{w}_{n}}{\Delta t} \cdot \Delta V_{n} .
$$

Equation (47) can be rewritten as

$$
a_{n(n-1)} p_{n-1}+a_{n n} p_{n}=b_{n}
$$

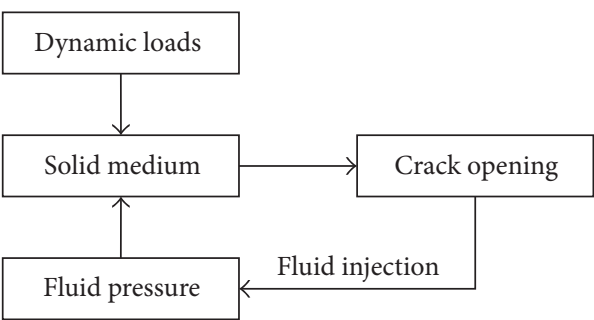

Figure 7: The coupling of the elasticity, fluid flow, and fracture growth.

where

$$
\begin{aligned}
& a_{n(n-1)}=\frac{k_{\mathrm{w}} A_{\mathrm{w}}}{L_{n-1}}, \\
& a_{n n}=-\frac{k_{\mathrm{w}} A_{\mathrm{w}}}{L_{n-1}}-\frac{k_{\mathrm{e}} A_{\mathrm{e}}}{L_{n}}, \\
& b_{n}=-\frac{\Delta \bar{w}_{n}}{\Delta t} \cdot \Delta V_{n}-k_{\mathrm{e}} A_{\mathrm{e}} \frac{p_{\mathrm{t}}}{L_{n}}, \\
& k_{\mathrm{w}}=\delta_{\mathrm{w}} k_{n-1}+\left(1-\delta_{\mathrm{w}}\right) k_{n}, A_{\mathrm{w}}=\delta_{\mathrm{w}} A_{n-1}+\left(1-\delta_{\mathrm{w}}\right) A_{n} .
\end{aligned}
$$

$k_{\mathrm{e}}$ and $A_{\mathrm{e}}$ can be calculated directly according to crack width at the fluid front, and $p_{\mathrm{t}}$ is the pressure of the fluid front and $p_{\mathrm{t}}=0$.

By setting up discretised equations of the forms (42), (45), and (48), at each node, the fluid pressure distribution along a crack can be obtained.

3.3. Coupling Scheme. To correctly solve the system of equations, the elasticity, fluid flow, and fracture growth should be coupled together; see Figure 7. The crack opening causes the fluid injecting into the solid medium. The injecting fluid produces fluid pressures along crack surface and affects the deformation of the solid medium and crack propagation. Due to the deformation of the solid medium, the crack opening changes again.

According to (36), the relations between the fluid pressure and crack width are nonlinear. It is necessary to use an algorithm that can solve iteratively the fluid pressure and crack width. The staggered Newton algorithm [40] is used here. The iteration processes for solving fluid pressure at time $t$ are stated as follows:

(i) Assume that the fluid pressure $p_{k}$ has been computed at the $k$ th step iteration. Compute dynamic responses of solid medium under dynamic loads and fluid pressure by using XFEM. According to dynamic responses, we can obtain the crack width $w_{k}$ at the $k$ th step iteration.

(ii) According to the known crack width $w_{k}$ at the $k$ th step iteration, compute the fluid pressure $p_{k+1 / 2}$ by using FVM.

(iii) Compute the fluid pressure $p_{k+1}$ at the $(k+1)$ th step iteration by the flowing expression:

$$
p_{k+1}=(1-\alpha) p_{k}+\alpha p_{k+1 / 2} .
$$



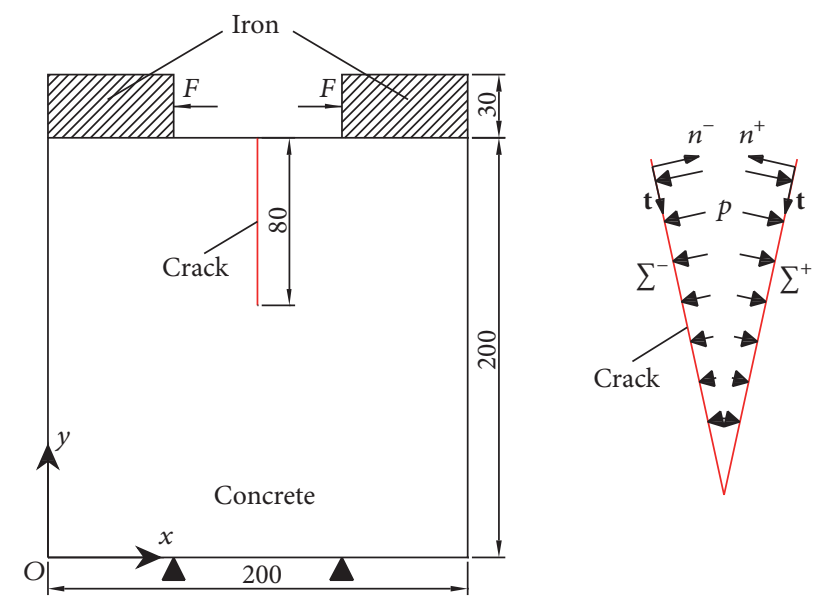

FIGURE 8: Schematic of a notched cubic concrete specimen subjected to a splitting force (unit: $\mathrm{mm}$ ).

(iv) Compute dynamic responses of solid medium under dynamic loads and fluid pressure $p_{k+1}$ by using XFEM. According to dynamic responses, we can obtain the crack width $w_{k+1 / 2}$.

(v) Compute the crack width $w_{k+1}$ at the $(k+1)$ th step iteration by the flowing expression:

$$
w_{k+1}=(1-\alpha) w_{k}+\alpha w_{k+1 / 2} .
$$

(vi) Given tolerance $\varepsilon=0.01$, compute the following expression:

$$
\delta p=\frac{\sum_{i=1}^{n}\left|p_{k+1}-p_{k}\right|}{\sum_{i=1}^{n}\left|p_{k+1}\right|}
$$

If $\delta p \leq \varepsilon$, finish iteration, or else go to (ii).

\section{Numerical Example}

As shown in Figure 8, a notched cubic concrete specimen with the dimension of $200 \mathrm{~mm} \times 200 \mathrm{~mm} \times 200 \mathrm{~mm}$ subjected to a splitting force was considered in this example. Here, the splitting force was applied on the iron. The iron was fastened on the concrete specimen. The material properties of concrete were Young modulus $E=36 \mathrm{GPa}$, Poisson ratio $v=0.167$, and mass density $\rho=2400 \mathrm{~kg} / \mathrm{m}^{3}$. The material properties of iron were Young modulus $E=200 \mathrm{GPa}$, Poisson ratio $v=0.3$, and density $\rho=7800 \mathrm{~kg} / \mathrm{m}^{3}$. In numerical model, the specimen was discretised into 10154 elements and 10370 nodes; see Figure 9. The concrete material's initiation toughness $K_{\text {Ic }}^{\text {ini }}$ equalled $0.888 \mathrm{MPa} \cdot \mathrm{m}^{1 / 2}$ for slow loading case and $0.909 \mathrm{MPa} \cdot \mathrm{m}^{1 / 2}$ for fast loading case, and its unstable fracture toughness $K_{\mathrm{Ic}}^{\mathrm{un}}$ equalled $2.914 \mathrm{MPa} \cdot \mathrm{m}^{1 / 2}$ for slow loading case and $3.028 \mathrm{MPa} \cdot \mathrm{m}^{1 / 2}$ for fast loading case. The parameters were tested by experiment. For the slow loading case, we set the time step $\Delta t=10 \mathrm{~s}$, while $\Delta t=0.1 \mathrm{~s}$ for the fast loading case.

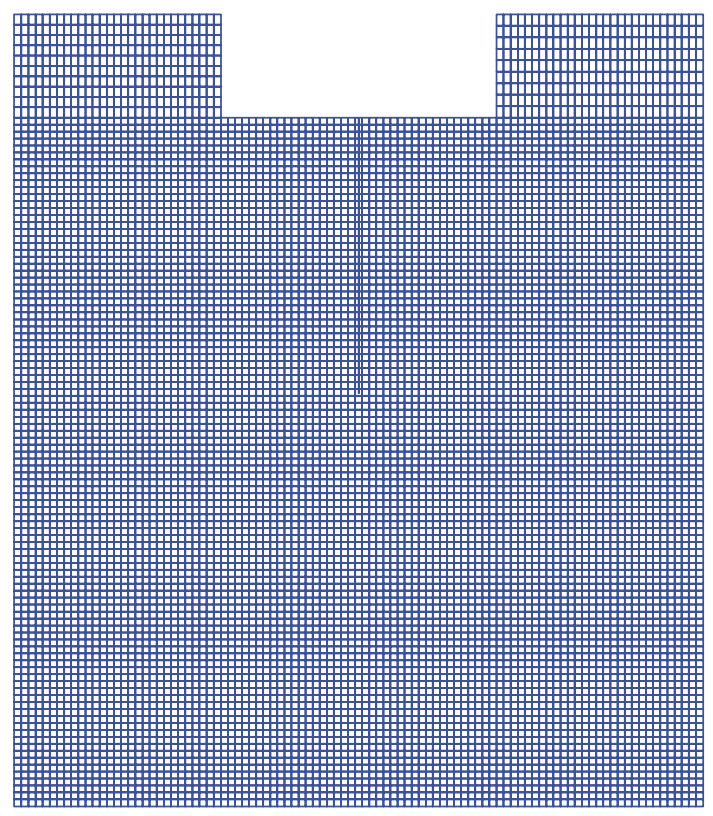

Figure 9: XFEM mesh.

In this section, we mainly investigated the water pressure distribution along crack surface and the effect of water pressure distribution on the fracture property.

4.1. Splitting Force versus CMOD Curve: Comparison with Our Experiments [41]. As shown in Figures 10 and 11, we plotted splitting force versus CMOD curve for slow loading case and fast loading case, respectively, with three different water pressure cases: (i) without water pressure; (ii) water pressure $p=0.2 \mathrm{MPa}$ at the crack mouth; (iii) water pressure $p=0.4 \mathrm{MPa}$ at the crack mouth. We also compared our present numerical results with our experimental results.

Both numerical and experimental results show that, (i) with the increase of the applied water pressure at the crack mouth, the peak value of the splitting force decreased dramatically; (ii) under the same case, compared with the slow loading case, the maximum splitting force from the fast loading case had an obvious increase. Additionally, a fairly satisfactory agreement can still be observed for the curve in ascending section. This verification indicated that the proposed model was quite effective for simulating hydraulic fracture problems.

We also listed the maximum splitting force with three different water pressure cases in Table 1 . The maximum error reached to $16.98 \%$.

\subsection{Effect of Water Pressure at the Crack Mouth on the Fracture} Property. For experiment, the maximum water pressure $p_{n}$ $=0.4 \mathrm{MPa}$ at the crack mouth was applied. Due to the limitation of experimental conditions, it was quite difficult in the experiment to increase the water pressure at the crack mouth for investigating its effect on the fracture property. The numerical simulation showed its huge advantages in extending test conditions. Here, we observed numerically 
TABLE 1: The maximum splitting force $F_{\max } / \mathrm{kN}$.

\begin{tabular}{|c|c|c|c|c|c|c|}
\hline & \multicolumn{3}{|c|}{ Slow loading case } & \multicolumn{3}{|c|}{ Fast loading case } \\
\hline & $p_{n}=0.0$ & $p_{n}=0.2 \mathrm{MPa}$ & $p_{n}=0.4 \mathrm{MPa}$ & $p_{n}=0.0 \mathrm{MPa}$ & $p_{n}=0.2 \mathrm{MPa}$ & $p_{n}=0.4 \mathrm{MPa}$ \\
\hline Numerical results & 15.60 & 13.31 & 10.75 & 19.51 & 17.02 & 14.00 \\
\hline Experimental results & 18.79 & 14.28 & 10.52 & 19.82 & 16.16 & 12.95 \\
\hline Error & $16.98 \%$ & $6.79 \%$ & $2.19 \%$ & $1.56 \%$ & $5.32 \%$ & $8.11 \%$ \\
\hline
\end{tabular}

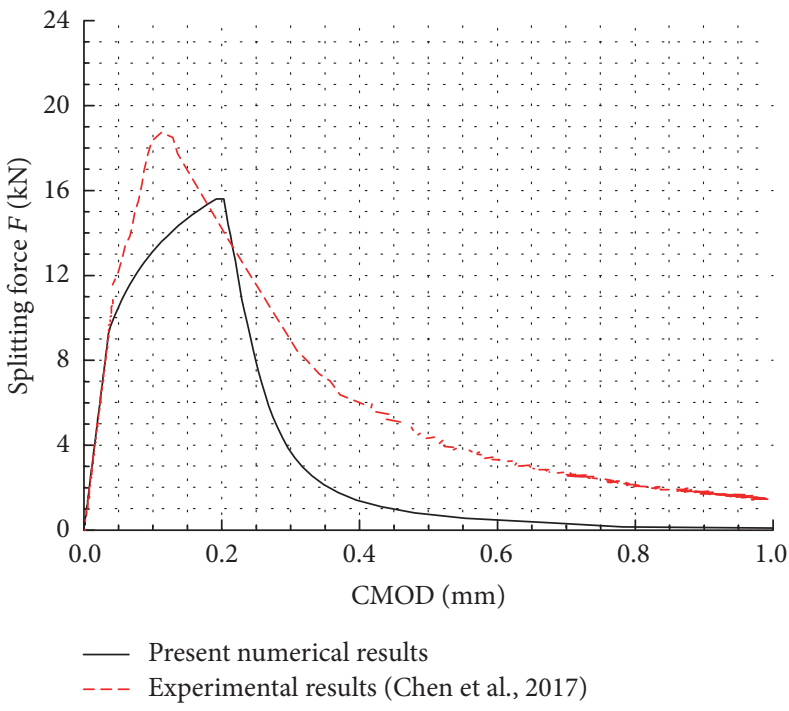

(a) Without water pressure

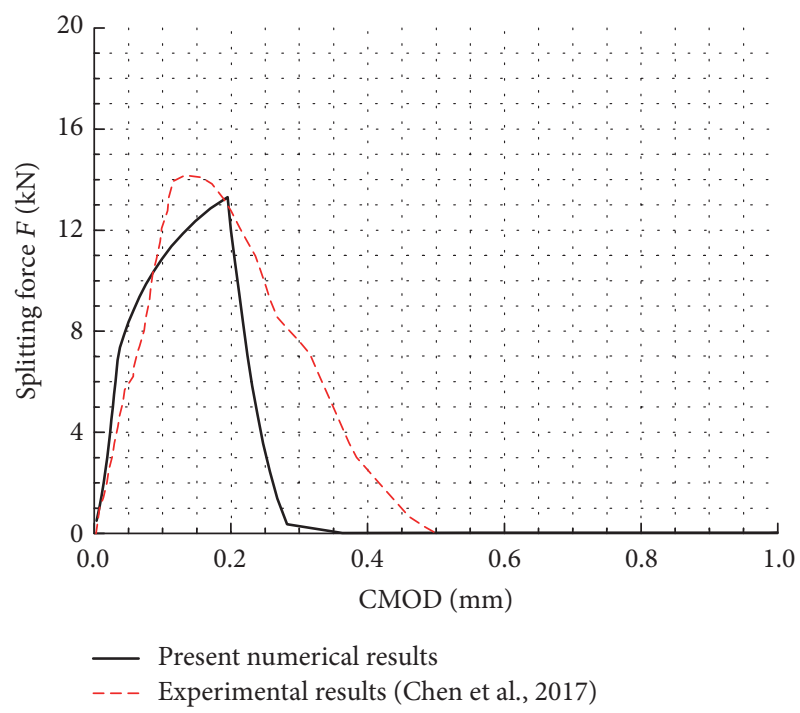

(b) Water pressure $p_{n}=0.2 \mathrm{MPa}$ at the crack mouth

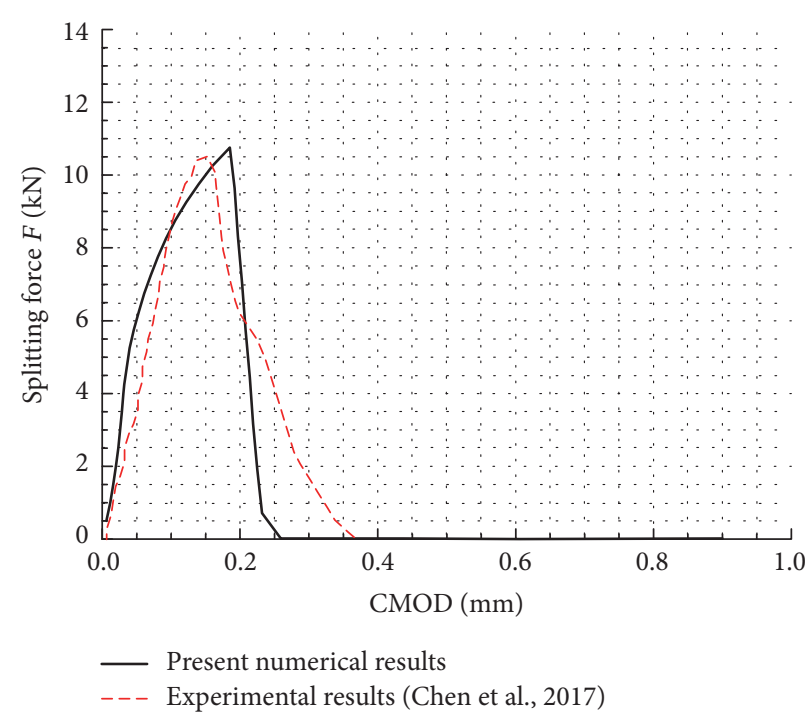

(c) Water pressure $p_{n}=0.4 \mathrm{MPa}$ at the crack mouth

FIGURE 10: Splitting force versus CMOD curve for slow loading case with different water pressures.

six cases, $p_{n}=0.0,0.2 \mathrm{MPa}, 0.4 \mathrm{MPa}, 0.6 \mathrm{MPa}, 0.8 \mathrm{MPa}$, and 1.0 MPa.

Figure 12(a) showed the effect of water pressure distribution on the fracture property for a slow loading case with six different water pressure cases and Figure 12(b) for fast loading case. We also plotted maximum splitting force versus applied water pressure at the crack mouth curve; see Figure 13. As depicted in Section 4.1, the results shown in these figures were still straightforward. With the increase of the applied water pressure at the crack mouth, the peak value of the splitting force decreased dramatically. Under the same case, compared with the slow loading case, the maximum splitting force from the fast loading case had an obvious increase. It was obvious that if the applied water pressure increased, the mechanical splitting force was designed to decrease to maintain the same CMOD. In Figure 13, it also could be found that if 


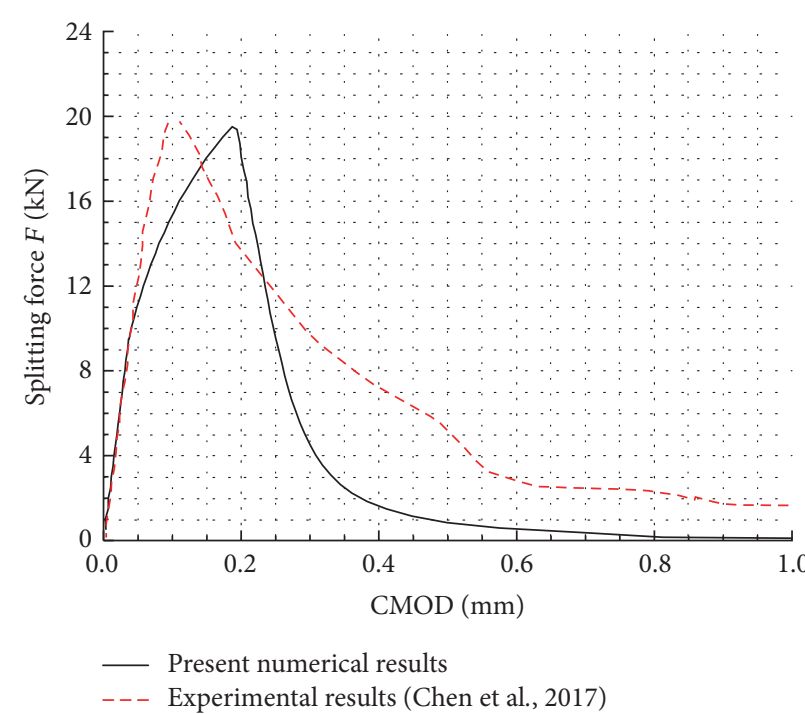

(a) Without water pressure

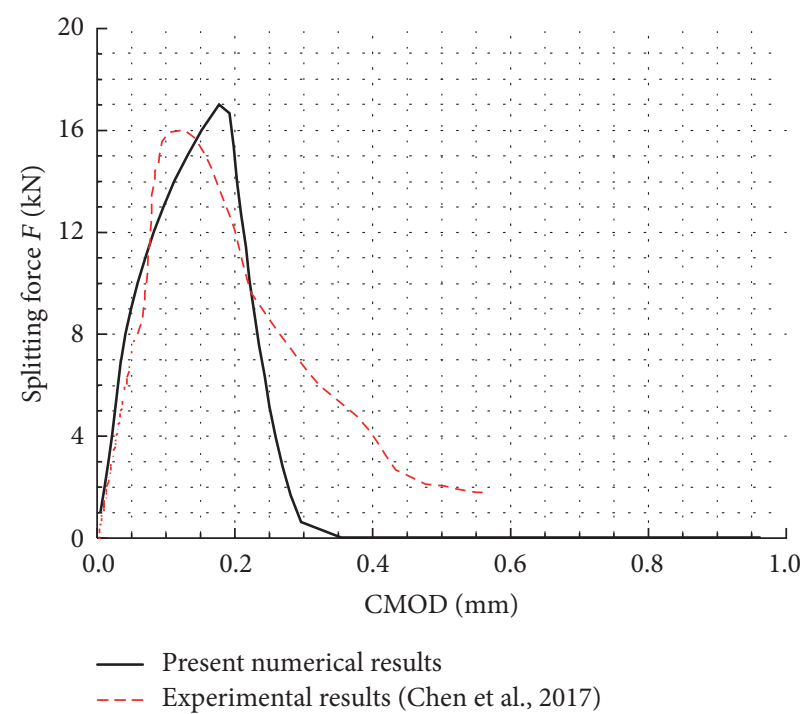

(b) Water pressure $p_{n}=0.2 \mathrm{MPa}$ at the crack mouth

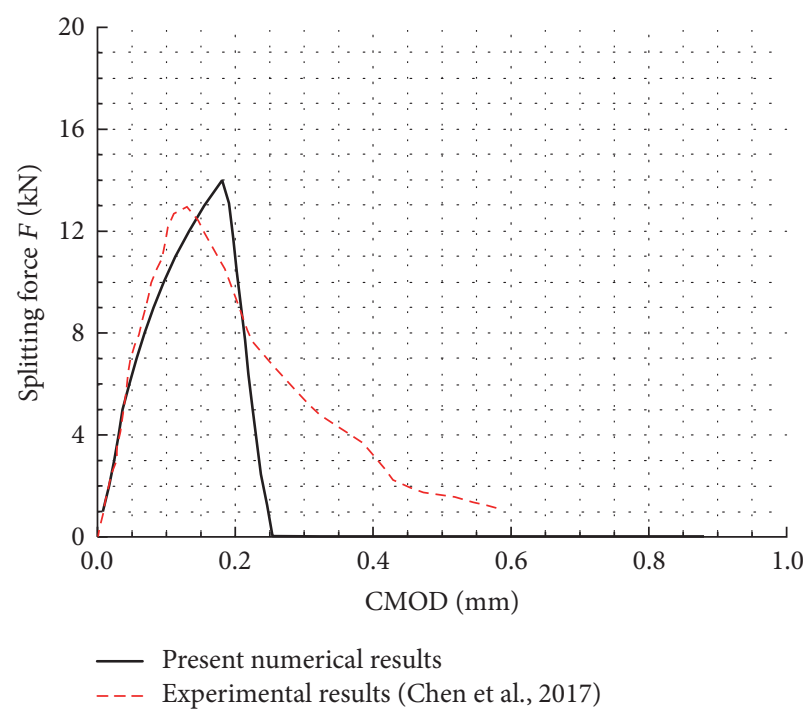

(c) Water pressure $p_{n}=0.4 \mathrm{MPa}$ at the crack mouth

FIGURE 11: Splitting force vesus CMOD curve for fast loading case with different water pressures.

$p_{n} \geq 0.6 \mathrm{MPa}$, the decrease velocity of the maximum splitting force slowed down.

The F-CMOD curve contained two phases: the prepeak/peak response and the postpeak response. The CMOD value responding to peak response was always less than $0.2 \mathrm{~mm}$. With the increase of the applied water pressure at the crack mouth, the CMOD value responding to peak response decreased significantly.

\subsection{Water Pressure Distribution along a Propagating Crack} Surface. Figure 14 showed the water pressure distribution along a propagating crack surface for slow and fast loading cases. To limit the length of this paper, only the case $p_{n}=$ $0.4 \mathrm{MPa}$ was shown here. It could be seen that the water pressure distribution along crack surface followed parabolic distribution. But, at the beginning, the water pressure distribution closely approximated linear distribution. At last, the water pressure for most crack segments approximated the applied water pressure at the crack mouth. Adjacent to tip, the water pressure dropped rapidly to zero.

4.4. Failure Patterns. As shown in Figures 15 and 16, we showed the failure mode for slow and fast loading cases with $p_{n}=0.2 \mathrm{MPa}$. In numerical model, we assumed that the concrete specimen was ideal homogeneous material. Therefore, the numerical results showed that the crack was pure mode-I crack and propagated in a straight manner. The experimental results also showed that the crack propagated approximately in a straight manner, but a slight deflection could still be observed. This phenomenon could be interpreted as the heterogeneity of the actual concrete specimen. 


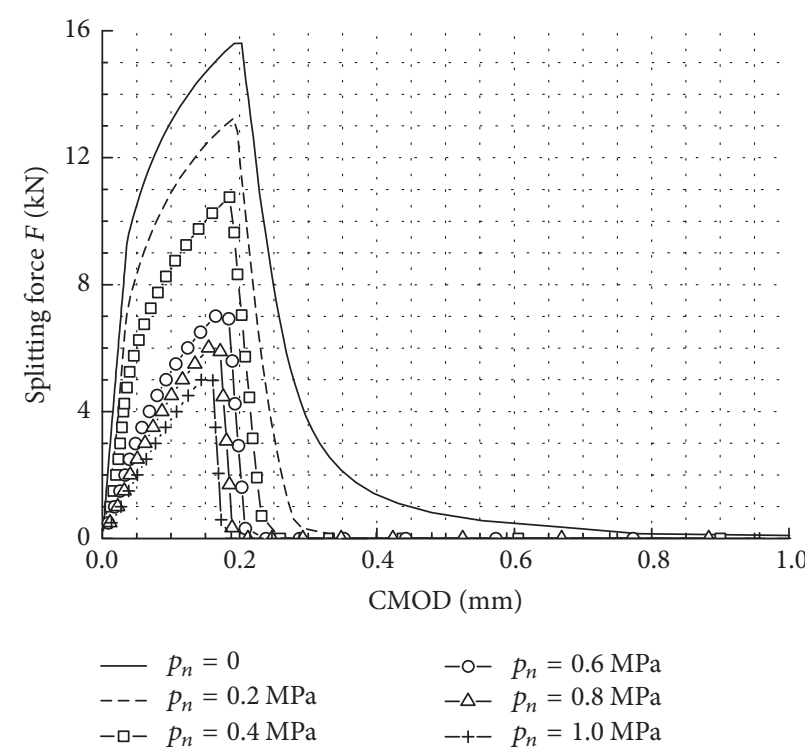

(a) Slow loading case

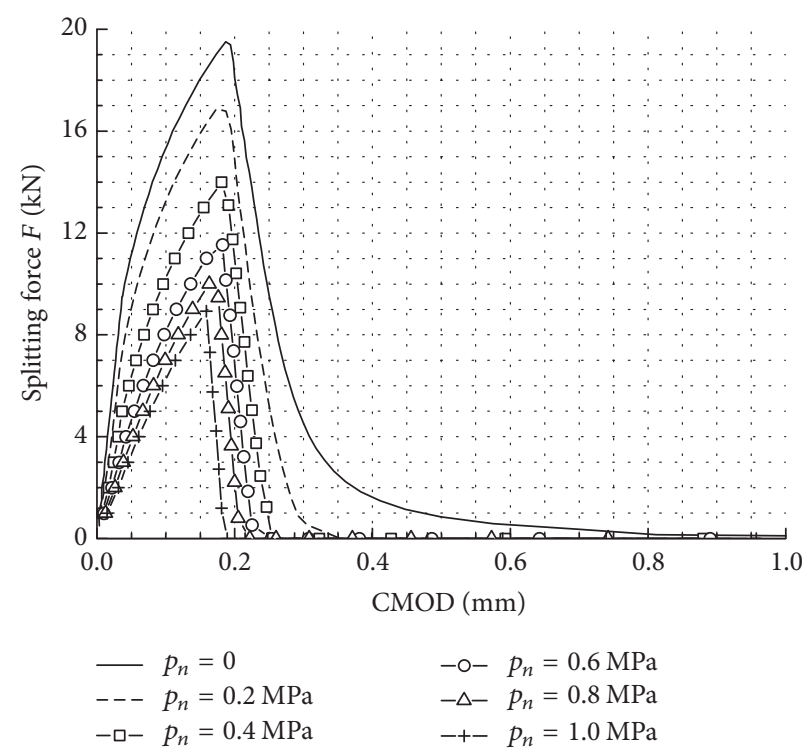

(b) Fast loading case

FIGURE 12: Effect of water pressure at the crack mouth on the fracture property.

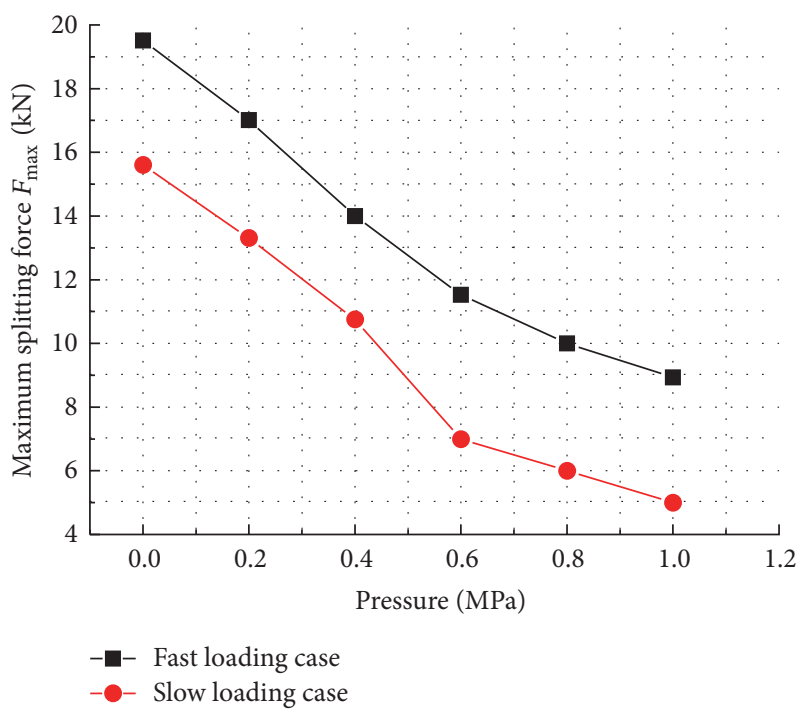

FIGURE 13: Maximum splitting force versus applied water pressure at the crack mouth curve.

\section{Summary and Conclusions}

In this paper, we model the fluid flow within the crack as onedimensional flow and assume that the flow is laminar; the fluid is incompressible and accounts for the time-dependent rate of crack opening. Here, we discretise the flow equation by finite volume methods. The extended finite element methods are used for solving solid medium with crack under dynamic loads. Having constructed the approximation of dynamic extended finite element methods, the derivation of governing equation for dynamic extended finite element methods is presented. The implicit time algorithm is elaborated for the time descritisation of dominant equation. In addition, the interaction integral method is given for evaluating stress intensity factors. Then, the coupling model for modelling hydraulic fracture can be established by the extended finite element methods and the finite volume methods. We compare our present numerical results with our experimental results for verifying the proposed model. Finally, we investigate the water pressure distribution along crack surface and the effect of water pressure distribution on the fracture property. Some valuable conclusions can be drawn from this study.

(i) Some conclusions between numerical results and experimental results were quite identical. A fairly satisfactory agreement could also be observed for FCMOD curve in ascending section. Therefore, the proposed model was quite effective for simulating hydraulic fracture problems.

(ii) The F-CMOD curve contained two phases: the prepeak/peak response and the postpeak response. The CMOD value responding to peak response was always less than $0.2 \mathrm{~mm}$. With the increase of the applied water pressure at the crack mouth, the CMOD value responding to peak response decreased significantly.

(iii) With the increase of the applied water pressure at the crack mouth, the peak value of the splitting force decreased dramatically. Under the same case, compared with the slow loading case, the maximum splitting force from the fast loading case had an obvious increase.

(iv) The water pressure distribution along crack surface followed parabolic distribution. But, at the beginning, the water pressure distribution closely approximated linear distribution. At last, the water pressure for most crack segments approximated the applied water pressure at the crack mouth. Adjacent to tip, the water pressure dropped rapidly to zero. 

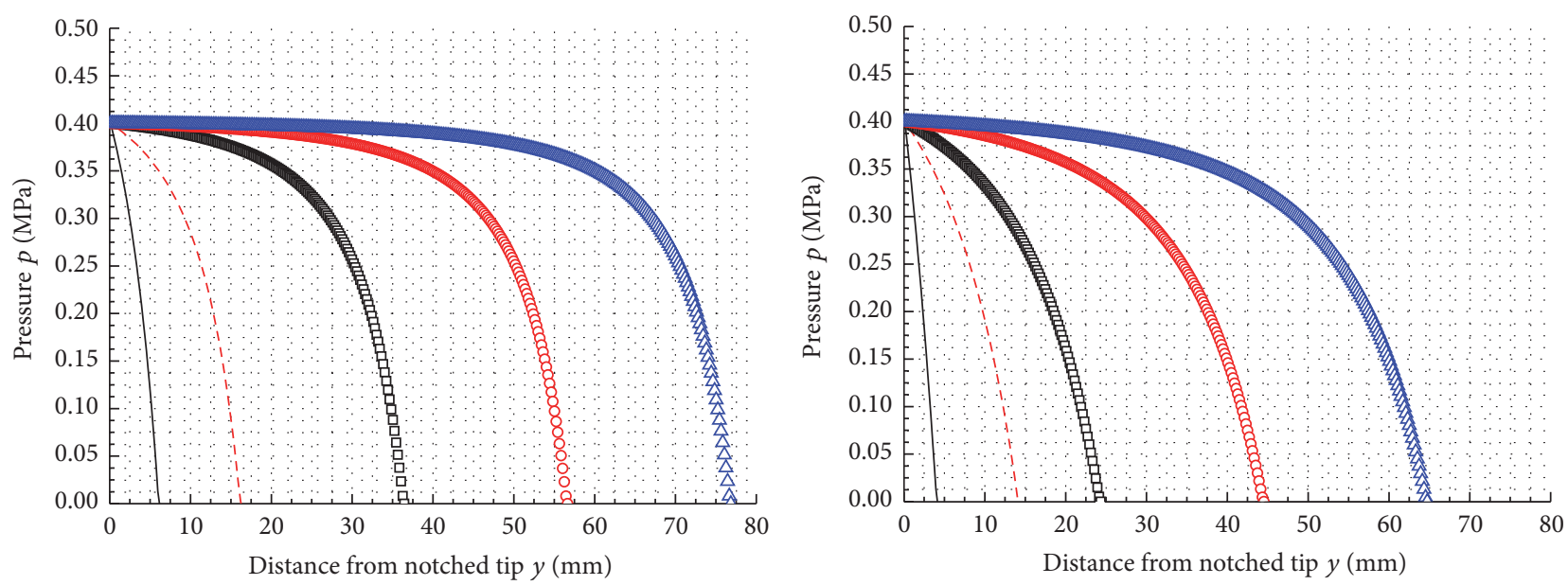

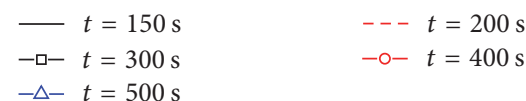

(a) Slow loading case

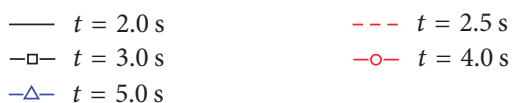

(b) Fast loading case

FIGURE 14: Water pressure distribution along a propagating crack surface.

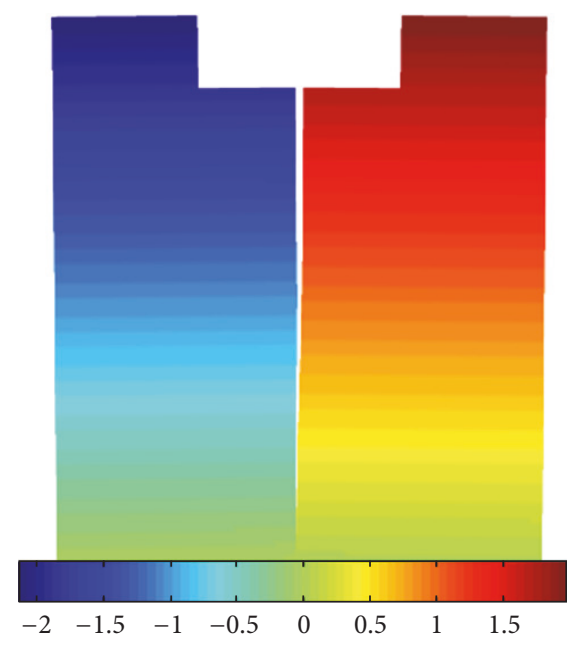

(a) $x$-directional displacement contours $/ \mathrm{mm}$

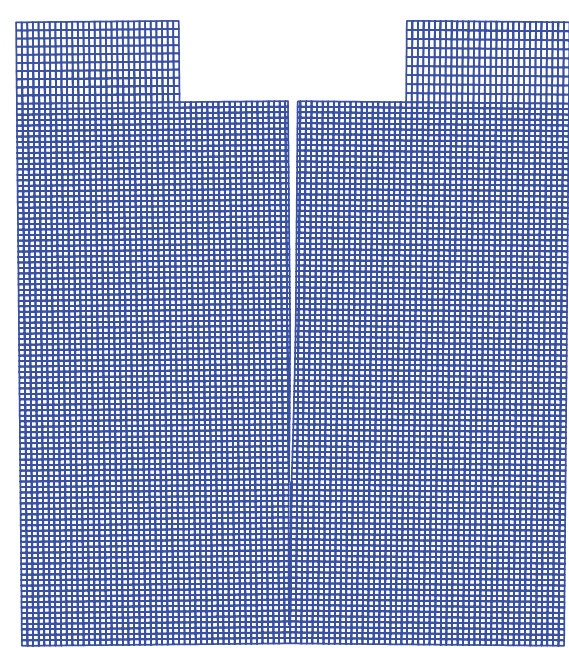

(c) Deformed mesh

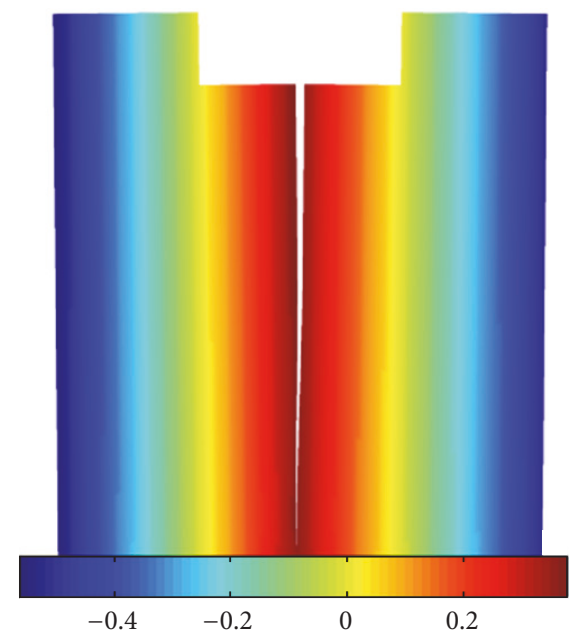

(b) $y$-directional displacement contours $/ \mathrm{mm}$

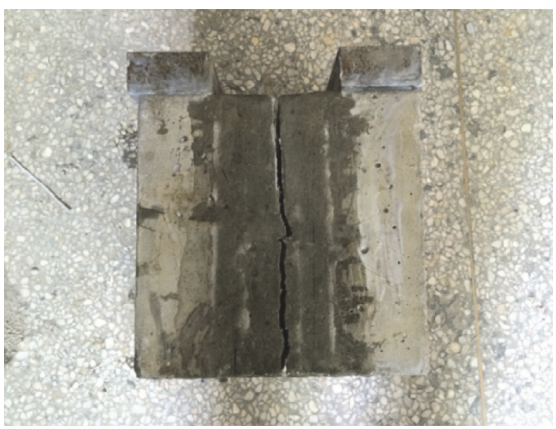

(d) Failure mode of experiment

FIGURE 15: Failure mode for slow loading case with $p_{n}=0.2 \mathrm{MPa}$. 


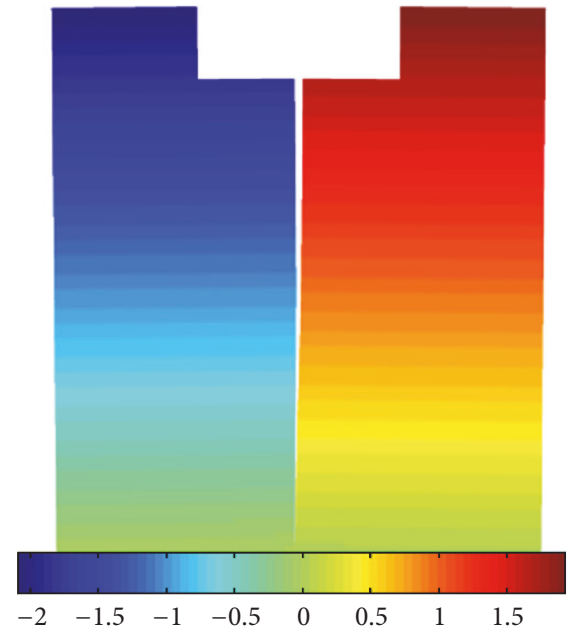

(a) $x$-directional displacement contours $/ \mathrm{mm}$

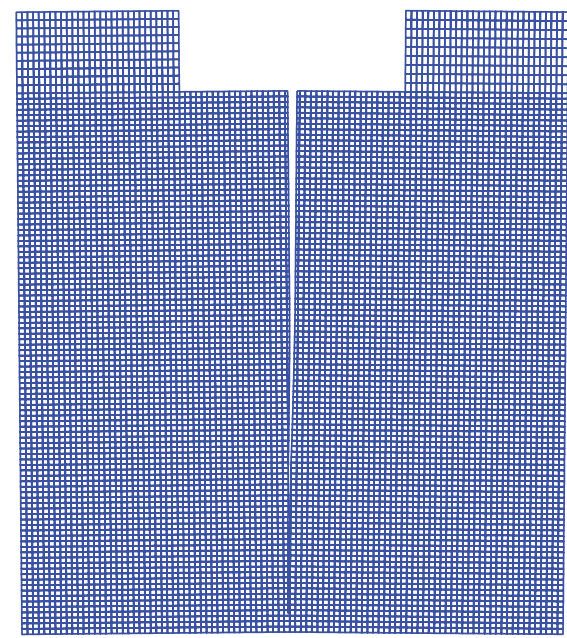

(c) Deformed mesh

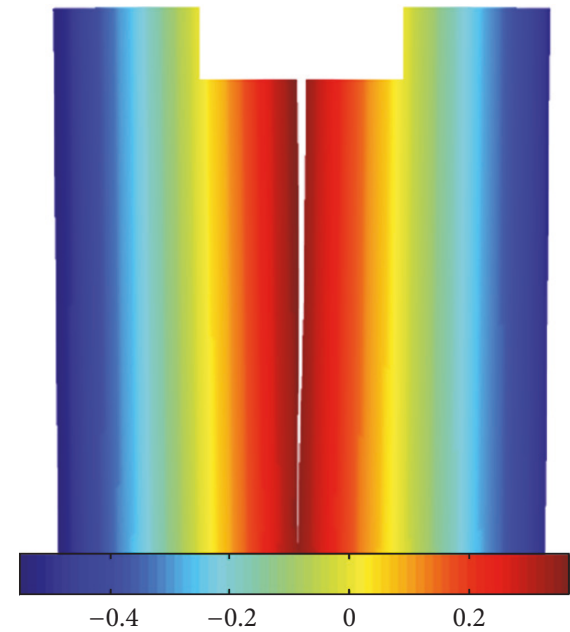

(b) $y$-directional displacement contours $/ \mathrm{mm}$

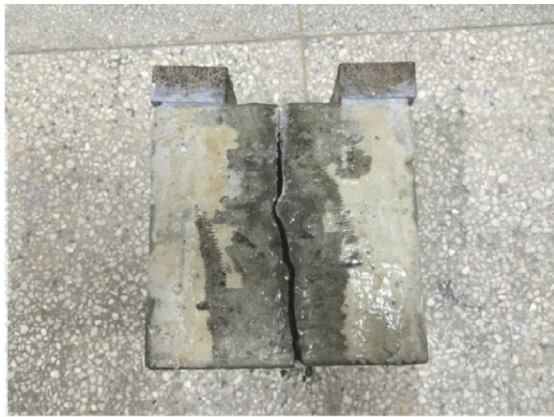

(d) Failure mode of experiment

FIgURE 16: Failure mode for fast loading case with $p_{n}=0.2 \mathrm{MPa}$.

(v) In numerical model, we assumed that the concrete specimen was ideal homogeneous material. Therefore, the numerical results showed that the crack was pure mode-I crack and propagated in a straight manner. The experimental results also showed that the crack propagated approximately in a straight manner, but a slight deflection could still be observed. This phenomenon could be interpreted as the heterogeneity of the actual concrete specimen.

Our future work will further investigate the water pressure distribution along a propagating crack surface and the effect of water pressure distribution on the fracture property with considering crack opening-closing.

\section{Conflicts of Interest}

The authors declare that there are no conflicts of interest regarding the publication of this paper.

\section{Acknowledgments}

The authors gratefully acknowledge support for this research from the National Natural Science Foundation of China (Grants nos. 51309088, 11372098, 51579084, and 51308188), the Fundamental Research Funds for the Central Universities (Grant no. 2015B01714), the National Sci-Tech Support Plan of China (Grant no. 2015BAB07B10), and China Postdoctoral Science Foundation funded project (Grant no. 2014T70466). The authors also would like to thank Professor Charles Augarde for the discussion during their visit to Durham University, who stayed in Hohai University supported by the Royal Society.

\section{References}

[1] E. Detournay, "Mechanics of Hydraulic Fractures," Annual Review of Fluid Mechanics, vol. 48, pp. 311-339, 2016.

[2] J. Adachi, E. Siebrits, A. Peirce, and J. Desroches, "Computer simulation of hydraulic fractures," International Journal of Rock Mechanics and Mining Sciences, vol. 44, no. 5, pp. 739-757, 2007. 
[3] A. Linkov, "Bench-mark solution for a penny-shaped hydraulic fracture driven by a thinning fluid," Physics, vol. 77, no. 77, pp. 14-23, 2015.

[4] A. P. Bunger and E. Detournay, "Early-time solution for a radial hydraulic fracture," Journal of Engineering Mechanics, vol. 133, no. 5, pp. 534-540, 2007.

[5] F. Javanmardi, P. Léger, and R. Tinawi, "Seismic structural stability of concrete gravity dams considering transient uplift pressures in cracks," Engineering Structures, vol. 27, no. 4, pp. 616-628, 2005.

[6] T. J. Boone and A. R. Ingraffea, "A numerical procedure for simulation of hydraulically-driven fracture propagation in poroelastic media," International Journal for Numerical and Analytical Methods in Geomechanics, vol. 14, no. 1, pp. 27-47, 1990.

[7] Z. Wu and L. N. Y. Wong, "Extension of numerical manifold method for coupled fluid flow and fracturing problems," International Journal for Numerical and Analytical Methods in Geomechanics, vol. 38, no. 18, pp. 1990-2008, 2014.

[8] A. Lisjak, P. Kaifosh, L. He, B. S. A. Tatone, O. K. Mahabadi, and G. Grasselli, "A 2D, fully-coupled, hydro-mechanical, FDEM formulation for modelling fracturing processes in discontinuous, porous rock masses," Computers \& Geosciences, vol. 81, pp. $1-18,2017$.

[9] U. Ohlsson, M. Nyström, T. Olofsson, and K. Waagaard, "Influence of hydraulic pressure in fracture mechanics modelling of crack propagation in concrete," Materials and Structures/Materiaux et Constructions, vol. 31, no. 207, pp. 203-208, 1996.

[10] F. Javanmardi, P. Léger, and R. Tinawi, "Seismic water pressure in cracked concrete gravity dams: Experimental study and theoretical modeling," Journal of Structural Engineering, vol. 131, no. 1, pp. 139-150, 2005.

[11] G. Bolzon and G. Cocchetti, "Direct assessment of structural resistance against pressurized fracture," International Journal for Numerical and Analytical Methods in Geomechanics, vol. 27, no. 5, pp. 353-378, 2003.

[12] J. M. Segura and I. Carol, "Numerical modelling of pressurized fracture evolution in concrete using zero-thickness interface elements," Engineering Fracture Mechanics, vol. 77, no. 9, pp. 1386-1399, 2010.

[13] J. Q. Bao, E. Fathi, and S. Ameri, "A coupled finite element method for the numerical simulation of hydraulic fracturing with a condensation technique," Engineering Fracture Mechanics, vol. 131, pp. 269-281, 2014.

[14] A. Shojaei, A. Dahi Taleghani, and G. Li, "A continuum damage failure model for hydraulic fracturing of porous rocks," International Journal of Plasticity, vol. 59, pp. 199-212, 2014.

[15] P. Gupta and C. A. Duarte, "Simulation of non-planar threedimensional hydraulic fracture propagation," International Journal for Numerical and Analytical Methods in Geomechanics, vol. 38, no. 13, pp. 1397-1430, 2014.

[16] S. Cherny, V. Lapin, D. Esipov et al., "Simulating fully 3D nonplanar evolution of hydraulic fractures," International Journal of Fracture, vol. 201, no. 2, pp. 181-211, 2016.

[17] L. Q. Choo, Z. Zhao, H. Chen, and Q. Tian, "Hydraulic fracturing modeling using the discontinuous deformation analysis (DDA) method," Computers \& Geosciences, vol. 76, pp. 12-22, 2016.
[18] X. Zhuang, J. Chun, and H. Zhu, "A comparative study on unfilled and filled crack propagation for rock-like brittle material," Theoretical and Applied Fracture Mechanics, vol. 72, pp. 110-120, 2014.

[19] G. Yi, T. Yu, T. Q. Bui, C. Ma, and S. Hirose, "SIFs evaluation of sharp V-notched fracture by XFEM and strain energy approach," Theoretical and Applied Fracture Mechanics, vol. 89, pp. 35-44, 2017.

[20] J. Shouyan, D. Chengbin, G. Chongshi, and C. Xiaocui, "XFEM analysis of the effects of voids, inclusions, and other cracks on the dynamic stress intensity factor of a major crack," Fatigue \& Fracture of Engineering Materials Structures, vol. 37, no. 8, pp. 866-882, 2014.

[21] J. Réthoré, R. de Borst, and M.-A. Abellan, "A two-scale approach for fluid flow in fractured porous media," International Journal for Numerical Methods in Engineering, vol. 71, no. 7, pp. 780-800, 2007.

[22] B. Lecampion, "An extended finite element method for hydraulic fracture problems," Communications in Numerical Methods in Engineering, vol. 25, no. 2, pp. 121-133, 2009.

[23] E. Gordeliy and A. Peirce, "Coupling schemes for modeling hydraulic fracture propagation using the XFEM," Computer Methods Applied Mechanics and Engineering, vol. 253, pp. 305322, 2013.

[24] E. Gordeliy and A. Peirce, "Implicit level set schemes for modeling hydraulic fractures using the XFEM," Computer Methods Applied Mechanics and Engineering, vol. 266, pp. 125-143, 2013.

[25] A. R. Khoei, M. Vahab, E. Haghighat, and S. Moallemi, "A meshindependent finite element formulation for modeling crack growth in saturated porous media based on an enriched-FEM technique," International Journal of Fracture, vol. 188, no. 1, pp. 79-108, 2014.

[26] A. D. Taleghani, Analysis of hydraulic fracture propagation in fractured reservoirs: an improved model for the interaction between induced and natural fractures, The University of Texas at Austin, 2009.

[27] S. Salimzadeh and N. Khalili, "A three-phase XFEM model for hydraulic fracturing with cohesive crack propagation," Computers \& Geosciences, vol. 69, pp. 82-92, 2015.

[28] K. Wang, Q. Zhang, X. Xia, L. Wang, and X. Liu, "Analysis of hydraulic fracturing in concrete dam considering fluidstructure interaction using XFEM-FVM model," Engineering Failure Analysis, vol. 57, pp. 399-412, 2015.

[29] G. K. Batchelor, Introduction to Fluid Dynamics, Cambridge University Press, Cambridge, UK, 1967.

[30] D. I. Garagash, "Propagation of a plane-strain hydraulic fracture with a fluid lag: Early-time solution," International Journal of Solids and Structures, vol. 43, no. 18-19, pp. 5811-5835, 2006.

[31] N. Moës, J. Dolbow, and T. Belytschko, "A finite element method for crack growth without remeshing," International Journal for Numerical Methods in Engineering, vol. 46, no. 1, pp. 131-150, 1999.

[32] G. Ventura, "On the elimination of quadrature subcells for discontinuous functions in the eXtended Finite-Element Method," International Journal for Numerical Methods in Engineering, vol. 66, no. 5, pp. 761-795, 2006.

[33] S. P. A. Bordas, T. Rabczuk, N.-X. Hung et al., "Strain smoothing in FEM and XFEM," Computers \& Structures, vol. 88, no. 23-24, pp. 1419-1443, 2010.

[34] S. Natarajan, D. R. Mahapatra, and S. P. Bordas, "Integrating strong and weak discontinuities without integration subcells 
and example applications in an XFEM/GFEM framework," International Journal for Numerical Methods in Engineering, vol. 83, no. 3, pp. 269-294, 2010.

[35] S. Natarajan, S. Bordas, and D. . Mahapatra, "Numerical integration over arbitrary polygonal domains based on SchwarzChristoffel conformal mapping," International Journal for Numerical Methods in Engineering, vol. 80, no. 1, pp. 103-134, 2009.

[36] M. Attigui and C. Petit, "Mixed-mode separation in dynamic fracture mechanics: New path independent integrals," International Journal of Fracture, vol. 84, no. 1, pp. 19-36, 1997.

[37] F. Erdogan and G. C. Sih, "On the crack extension in plates under plane loading and transverse shear," Journal of Basic Engineering, vol. 85, no. 4, pp. 519-527, 1963.

[38] N. Sukumar and J.-H. Prévost, "Modeling quasi-static crack growth with the extended finite element method Part I: Computer implementation," International Journal of Solids and Structures, vol. 40, no. 26, pp. 7513-7537, 2003.

[39] S. Xu and H. W. Reinhardt, "Determination of double- $K$ criterion for crack propagation in quasi-brittle fracture, part I: experimental investigation of crack propagation," International Journal of Fracture, vol. 98, no. 2, pp. 111-149, 1999.

[40] A. R. Khoei, M. Hirmand, M. Vahab, and M. Bazargan, "An enriched FEM technique for modeling hydraulically driven cohesive fracture propagation in impermeable media with frictional natural faults: Numerical and experimental investigations," International Journal for Numerical Methods in Engineering, vol. 104, no. 6, pp. 439-468, 2015.

[41] X. Chen, C. Du, M. You, S. Jiang, and L. Sun, "Experimental study on water fracture interactions in concrete," Engineering Fracture Mechanics, vol. 179, pp. 314-327, 2017. 


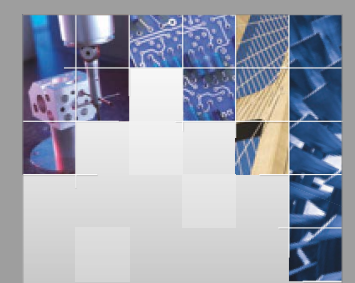

\section{Enfincering}
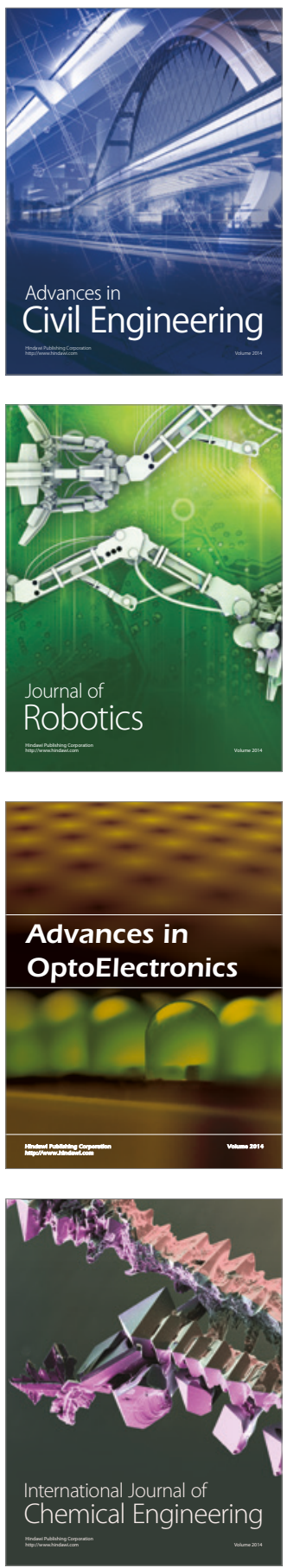

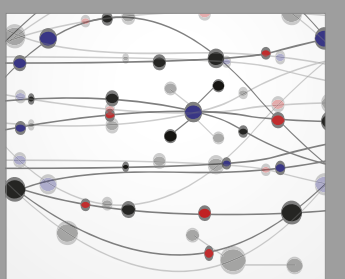

The Scientific World Journal

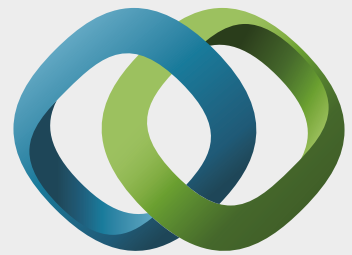

\section{Hindawi}

Submit your manuscripts at

https://www.hindawi.com
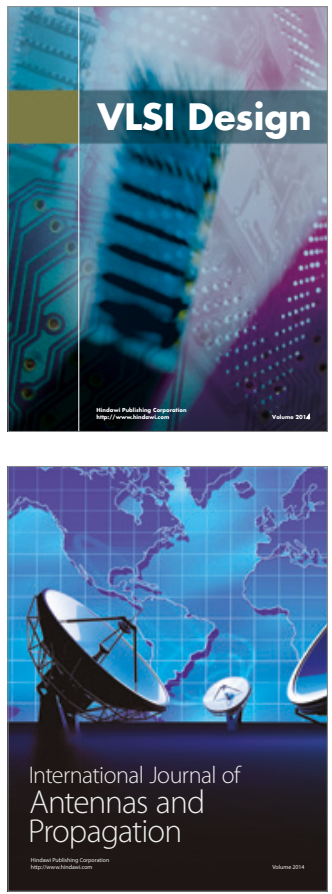

\section{Rotating}

Machinery
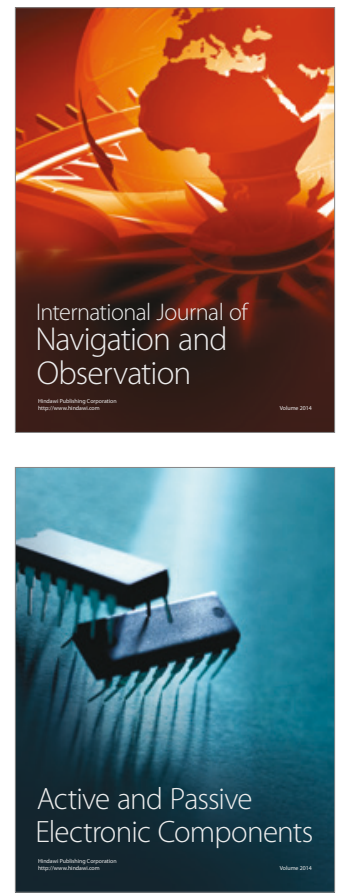
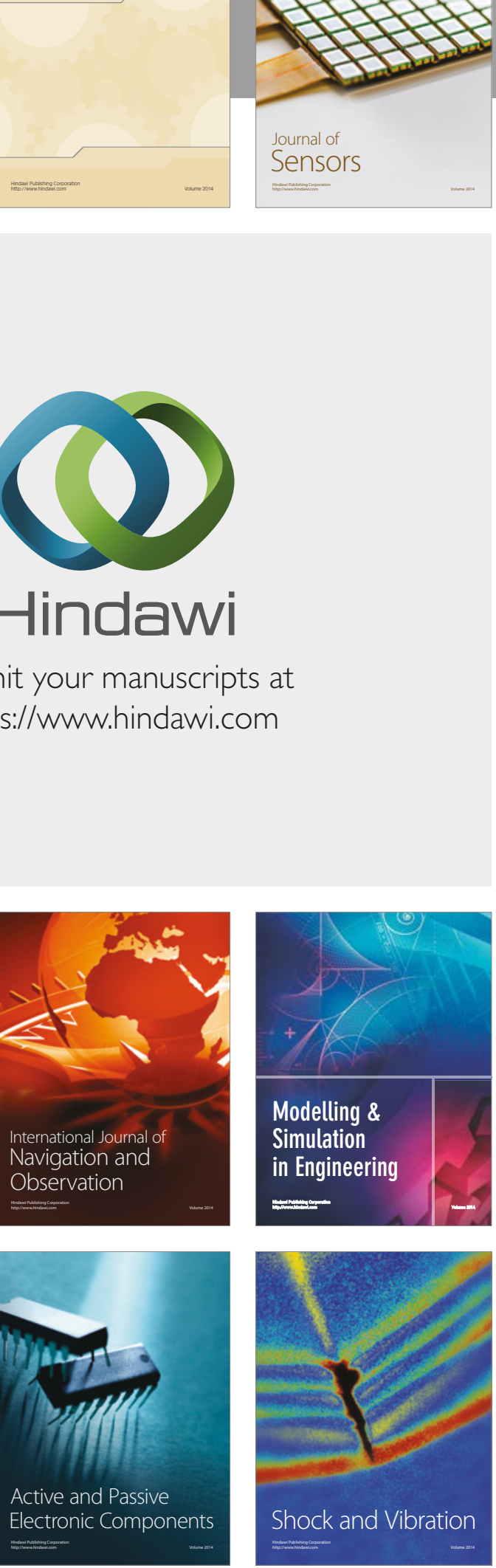
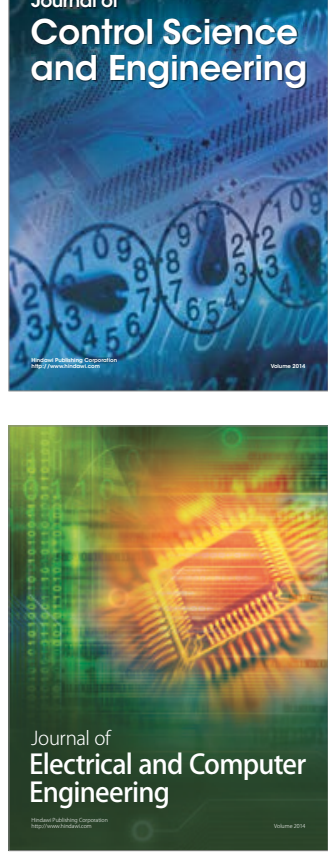

Distributed

Journal of

Control Science

and Engineering
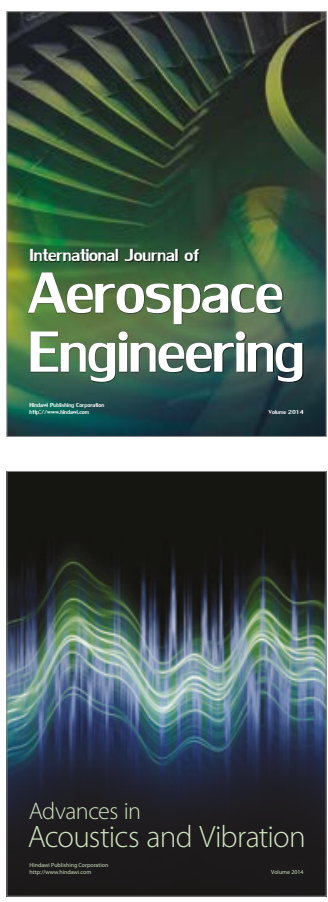

Sensor Networks 